\title{
SPINORIAL REPRESENTATION OF SURFACES IN LORENTZIAN HOMOGENEOUS SPACES OF DIMENSION 3
}

\author{
BERENICE ZAVALA JIMÉNEZ
}

\begin{abstract}
We find a spinorial representation of a Riemannian or Lorentzian surface in a Lorentzian homogeneous space of dimension 3. We in particular obtain a representation theorem for surfaces in the $\mathbb{L}(\kappa, \tau)$ spaces. We then recover the Calabi correspondence between minimal surfaces in $\mathbb{R}^{3}$ and maximal surfaces in $\mathbb{R}_{1}^{3}$, and obtain a new Lawson type correspondence between CMC surfaces in $\mathbb{R}_{1}^{3}$ and in the 3-dimensional pseudo-hyperbolic space $\mathbb{H}_{1}^{3}$.
\end{abstract}

MSC 2020: 53C40, 53C27, 53C50

Keywords: Isometric immersions, spin geometry, Lorentzian homogeneous spaces.

\section{INTRODUCTION}

A 3-dimensional Lorentzian homogeneous space, simply connected and complete, is a Lie group with a left-invariant Lorentzian metric, or one of the following symmetric spaces (see [9], [6], [7], [25],[26] and [27] for a detailed description of this classification):

- the Minkowski space $\mathbb{R}_{1}^{3}$, the pseudo-sphere $\mathbb{S}_{1}^{3}$ (the de Sitter space), the universal covering of the pseudo-hyperbolic space $\widetilde{\mathbb{H}_{1}^{3}}$ (the anti-de Sitter space);

- the products $\mathbb{R}_{-} \times \mathbb{S}^{2}, \mathbb{R}_{-} \times \mathbb{H}^{2}, \mathbb{R} \times \widetilde{\mathbb{S}_{1}^{2}}$ and $\mathbb{R} \times \widetilde{\mathbb{H}_{1}^{2}}$, where $\widetilde{\mathbb{S}_{1}^{2}}$ and $\widetilde{\mathbb{H}_{1}^{2}}$ are the universal coverings of the pseudo-sphere and the pseudo-hyperbolic space of dimension 2 ;

- the Cahen-Wallach spaces

$$
M_{c}:=\left(\mathbb{R}^{3}, g_{c}\right), g_{c(s, t, x)}:=2 d s d t+c x^{2} d s^{2}+d x^{2}, c= \pm 1 .
$$

Note that since the second fundamental group of a Lie group is necessarily trivial (see [24, Pag. 116-118]), in this list the symmetric spaces $\mathbb{S}_{1}^{3}$ and $\mathbb{R}_{-} \times \mathbb{S}^{2}$ do not admit a Lie group structure.

The aim of this paper is to characterize the immersion of a surface in a given simply connected complete Lorentzian homogeneous space of dimension 3 in terms of spinors. We will focus here on the Lorentzian metric Lie groups and on the symmetric spaces of constant curvature or which are a product, and leave the case of the Cahen-Wallach spaces to a further study. We will moreover pay a special attention to the family $\mathbb{L}(\kappa, \tau)$ of homogeneous spaces with a 4-dimensional group of isometries.

The similar problem in the Riemannian setting is entirely solved: Friedrich characterized in [11] the immersions in $\mathbb{R}^{3}$, Morel in [20] the immersions in $\mathbb{S}^{3}$ and $\mathbb{H}^{3}$ 
and Roth in [23] the immersions in Riemannian homogeneous spaces with a 4dimensional isometry group; we then found in [4] a characterization of the immersions in Riemannian metric Lie groups (of arbitrary dimension and co-dimension): since a simply connected Riemannian homogeneous space of dimension 3 is isometric to a metric Lie group or is the product manifold $\mathbb{S}^{2} \times \mathbb{R}$ (see $[18,19]$ ), we could achieve the characterization in the 3 -dimensional Riemmanian homogeneous spaces.

Besides the natural question of the extension to the Lorentzian setting of results obtained in Riemannian geometry, our motivation is to understand natural geometric correspondences between CMC surfaces in some Riemmanian or Lorentzian 3-dimensional homogeneous manifolds, as for instance the Lawson-type correspondences described in [10] and their Lorentzian analogues: to that end the spinorial approach seems promising since it permits to obtain a very concise formalism for the surface theories in these spaces. In that direction, we give at the end of the paper a Lawson-type correspondence between CMC surfaces in $\mathbb{R}_{1}^{3}$ and in $\mathbb{H}_{1}^{3}$, and we find a simple new proof of the correspondence between minimal surfaces in $\mathbb{R}^{3}$ and maximal surfaces in $\mathbb{R}_{1}^{3}$.

Let us quote some papers concerning immersions in 3-dimensional semi-Riemannian manifolds: Lawn $[12,13]$ studied immersions in $\mathbb{R}_{1}^{3}$, Lawn and Roth [14] in 3dimensional space forms and Roth [22] in Lorentzian products, especially in $M^{2}(k) \times$ $\mathbb{R}_{-}$. Bayard [1], Bayard and Patty [3] and Patty [21] obtained afterwards similar results in 3-dimensional Lorentzian space forms as a consequence of their study of immersions in dimension 4 . We will recover some of these results using explicitly the metric Lie group structures.

The paper is organized as follows: we present the spinorial representation of a Riemannian or a Lorentzian surface in a Lorentzian metric Lie group of dimension 3 in Sections 2 (via spinors of the group) and 3 (via spinors of the surface). We then study the immersions in Lorentzian products which have a group structure in Section 4, and in $\mathbb{S}_{1}^{3}$ and $\mathbb{H}_{1}^{3}$ in Section 5 . We then study the product $\mathbb{R}_{-} \times \mathbb{S}^{2}$ in Section 6 and the $\mathbb{L}(\kappa, \tau)$ spaces in Section 7 . We finally describe in Section 8 a Lawson-type correspondence between CMC surfaces in $\mathbb{R}_{1}^{3}$ and in $\mathbb{H}_{1}^{3}$, and give a spinorial proof of the correspondence between minimal surfaces in $\mathbb{R}^{3}$ and maximal surfaces in $\mathbb{R}_{1}^{3}$. A short appendix ends the paper, first on the identification of intrinsic and extrinsic spinor bundles, and then on the representation of skewsymmetric operators using the Clifford algebra.

\section{Representation of a surface into a metric Lie group}

2.1. Spinor bundle of a Lie group $G$. Let $G$ be a simply connected Lorentzian Lie group of dimension 3 endowed with a left-invariant metric $\langle\cdot, \cdot\rangle$ and $\mathfrak{g}$ its Lie algebra. The $\mathfrak{g}$-valued 1 -form

$$
\omega_{g}(v)=d L_{g^{-1}}(v), \quad v \in T_{g} G
$$

is the Maurer-Cartan form and we use it to give a trivialization of the tangent bundle of $G$

$$
\begin{aligned}
T G & \longrightarrow G \times \mathfrak{g} \\
X & \longmapsto\left(g, \omega_{g}(X)\right) .
\end{aligned}
$$


A section $X$ of $T G$ is a map $G \longrightarrow \mathfrak{g}$, and we will say that it is a left-invariant section if it is a constant function. Let us denote by $\bar{\nabla}$ the Levi-Civita connection of $G$ and by $\Gamma: \mathfrak{g} \longrightarrow \Lambda^{2} \mathfrak{g}$ the application which maps a left-invariant section $X \in \mathfrak{g}$ to the skew-symmetric linear transformation $\Gamma(X)$ of $\mathfrak{g}$ such that

$$
\bar{\nabla}_{X} Y=\Gamma(X)(Y)
$$

for all left-invariant section $Y \in \Gamma(T G)$. Using that the connection $\bar{\nabla}$ is torsion free, we have, for all $X, Y \in \mathfrak{g}$,

$$
\Gamma(X)(Y)-\Gamma(Y)(X)=[X, Y]
$$

Since the tangent bundle of $G$ is trivial, its orthonormal frame bundle is $Q_{G}=$ $G \times S O(\mathfrak{g})$ and $\widetilde{Q}_{G}=G \times \operatorname{Spin}(\mathfrak{g})$ is a spin structure over that bundle. The spin group has a natural representation in the Clifford algebra $C l(\mathfrak{g})$ given by the left-multiplication

$$
\begin{aligned}
\rho: \operatorname{Spin}(\mathfrak{g}) & \longrightarrow G l(C l(\mathfrak{g})) \\
x & \longmapsto \rho(x): C l(\mathfrak{g}) \longrightarrow C l(\mathfrak{g})
\end{aligned}
$$

We define the spinor bundle

$$
\Sigma G:=\widetilde{Q}_{G} \times{ }_{\rho} C l(\mathfrak{g}),
$$

associated to the spin structure $\widetilde{Q}_{G}$ (we identify $(p, q)$ and $\left(p^{\prime}, q^{\prime}\right)$ belonging to $\widetilde{Q}_{G} \times C l(\mathfrak{g})$ if there exists $g \in \operatorname{Spin}(\mathfrak{g})$ such that $\left.\left(p^{\prime}, q^{\prime}\right)=\left(p \cdot g, \rho\left(g^{-1}\right) q\right)\right)$. Note that this is not the usual spinor bundle since the representation $\rho$ is not the usual spin representation (it is not irreducible and not complex).

Remark 1. Since $\widetilde{Q}_{G}=G \times \operatorname{Spin}(\mathfrak{g})$ is the trivial spin structure there is a natural isomorphism between the spinor bundle defined in (3) and the trivial bundle $G \times$ $C l(\mathfrak{g})$.

Using the adjoint representation

$$
\operatorname{Spin}(\mathfrak{g}) \stackrel{A d}{\longrightarrow} S O(\mathfrak{g}) \longrightarrow G l(C l(\mathfrak{g}))
$$

we define as usual the Clifford bundle

$$
C l_{\Sigma G}:=\widetilde{Q} \times_{A d} C l(\mathfrak{g})
$$

and the Clifford action $\cdot: C l_{\Sigma G} \times \Sigma G \longrightarrow \Sigma G$.

In a slightly different context, the covariant derivative on $\Sigma G$ is defined in $[5$, Thm. 2.7] as follows: if $\varphi$ is a section of $\Sigma G, X \in T G$ and $\partial$ is the usual derivative in the trivialization of Remark 1, then the covariant derivative is

$$
\nabla_{X}^{G} \varphi=\partial_{X} \varphi+\frac{1}{2} \sum_{1 \leq k<l \leq 3} \varepsilon_{k} \varepsilon_{l}\left\langle\bar{\nabla}_{X} e_{k}, e_{l}\right\rangle e_{k} \cdot e_{l} \cdot \varphi,
$$

where $\left(e_{1}, e_{2}, e_{3}\right)$ is an orthonormal frame of $G, \varepsilon_{j}=\left\langle e_{j}, e_{j}\right\rangle= \pm 1$ and $\bar{\nabla}$ is the Levi-Civita connection in $G$.

Let us call a section $\varphi$ of $\Sigma G$ a left-invariant spinor field if it is constant when regarded as a function from $G$ to $C l(\mathfrak{g})$. By (4) the covariant derivative of a leftinvariant spinor field $\varphi$ is

$$
\nabla_{X}^{G} \varphi=\frac{1}{2} \Gamma(X) \cdot \varphi
$$


where $\Gamma(X) \in \Lambda^{2} \mathfrak{g} \subset C l(\mathfrak{g})$ is defined in (2) and · denotes the Clifford action.

2.2. The twisted spinor bundle over a surface. Let $(M, g)$ be a pseudoRiemannian surface of signature $\left(r_{1}, s_{1}\right)$, where $\left(r_{1}, s_{1}\right)=(0,2)$ if $M$ is Riemannian and $\left(r_{1}, s_{1}\right)=(1,1)$ if $M$ is Lorentzian. We suppose that $M$ is orientable in the Riemannian case, and orientable in space and in time in the Lorentzian case. Let us consider the trivial vector bundle $E=M \times \mathbb{R}$ endowed with a metric of signature $\left(r_{2}, s_{2}\right)$ in each fiber, with

$$
\left(r_{1}, s_{1}\right)+\left(r_{2}, s_{2}\right)=(1,2) .
$$

So the metric on $E$ is $-d \nu^{2}$ if $M$ is Riemannian and $+d \nu^{2}$ if $M$ is Lorentzian. Let $\widetilde{Q}_{M}$ and $\widetilde{Q}_{E}$ be spin structures in $M$ and $E$. Since the orthonormal frame bundle of $E$ is $Q_{E}=M \times\{1\}$ we have that $\widetilde{Q}_{E} \longrightarrow M$ is the trivial double covering with fiber $\{1,-1\}$. Let us now define the spinor bundles $\Sigma M$ and $\Sigma E$. For $k=1,2$ consider the following representations of the spin group

$$
\begin{aligned}
& \rho_{k}: \operatorname{Spin}\left(r_{k}, s_{k}\right) \longrightarrow \operatorname{Gl}\left(C l_{r_{k}, s_{k}}\right) \\
& x \longmapsto \rho_{k}(x): C l_{r_{k}, s_{k}} \longrightarrow C l_{r_{k}, s_{k}}
\end{aligned}
$$

and the spinor bundles over $M$ and $E$

$$
\Sigma M:=\widetilde{Q}_{M} \times \rho_{\rho_{1}} C l_{r_{1}, s_{1}} \text { and } \quad \Sigma E:=\widetilde{Q}_{E} \times_{\rho_{2}} C l_{r_{2}, s_{2}} .
$$

Since the algebras $C l_{r_{1}, s_{1}} \widehat{\otimes} C l_{r_{2}, s_{2}}$ and $C l_{r_{1}+r_{2}, s_{1}+s_{2}}$ are isomorphic, the vector bundle $\Sigma M \otimes \Sigma E$ is isomorphic to

$$
\Sigma:=\left(\widetilde{Q}_{M} \times_{M} \tilde{Q}_{E}\right) \times_{\rho} C l_{1,2},
$$

where

$$
\begin{aligned}
\rho: \operatorname{Spin}\left(r_{1}, s_{1}\right) \times \operatorname{Spin}\left(r_{2}, s_{2}\right) & \longrightarrow G l\left(C l_{1,2}\right) \\
x=\left(g_{1}, g_{2}\right) & \longmapsto \rho(x): C l_{1,2} \quad \longrightarrow \quad C l_{1,2}
\end{aligned}
$$

(the representation $\rho$ is equivalent to $\rho_{1} \otimes \rho_{2}$ ). We interpret the sub-bundle

$$
U \Sigma:=\left(\widetilde{Q}_{M} \times_{M} \tilde{Q}_{E}\right) \times_{\rho} \operatorname{Spin}(1,2) \quad \subset \quad \Sigma
$$

as the bundle of unit spinors. From the adjoint representation

$$
\operatorname{Spin}(2) \times \operatorname{Spin}(1) \longrightarrow \operatorname{Spin}(1,2) \stackrel{A d}{\longrightarrow} S O(1,2) \longrightarrow G l\left(C l_{1,2}\right)
$$

we have as usual the Clifford bundle

$$
C l_{\Sigma}:=\widetilde{Q} \times_{A d} C l_{1,2}
$$

and the Clifford action $\cdot: C l_{\Sigma} \times \Sigma \longrightarrow \Sigma$.

If $(M, g)$ is an orientable Riemannian (Lorentzian) surface isometrically immersed in $G$ and $E \rightarrow M$ is the normal bundle of $M$ in $G$ then $\Sigma G_{\mid M}=\Sigma=$ $\Sigma M \otimes \Sigma E$, and setting $\nabla:=\nabla^{M} \otimes \nabla^{E}$ where $\nabla^{M}$ and $\nabla^{E}$ are respectively the Levi-Civita and the normal connections we have the Gauss formula

$$
\nabla_{X} \varphi=-\frac{1}{2} \sum_{j=1}^{2} \varepsilon_{j} e_{j} \cdot B\left(X, e_{j}\right) \cdot \varphi+\nabla_{X}^{G} \varphi, \quad \varepsilon_{j}=g\left(e_{j}, e_{j}\right)= \pm 1
$$


for all $X \in T M$, where $B$ is the second fundamental form of the immersion and $\varphi$ is a section of $\left.\Sigma G\right|_{M}$. If $\varphi$ is moreover left-invariant, it follows from (5) that (7) is equivalent to

$$
\nabla_{X} \varphi=-\frac{1}{2} \sum_{j=1}^{2} \varepsilon_{j} e_{j} \cdot B\left(X, e_{j}\right) \cdot \varphi+\frac{1}{2} \Gamma(X) \cdot \varphi, \quad \varepsilon_{j}=g\left(e_{j}, e_{j}\right),
$$

for all $X \in T M$.

2.3. Definition and properties of $\langle\langle\cdot, \cdot\rangle\rangle$. In this section we define an application $\langle\langle\cdot, \cdot\rangle\rangle: \Sigma \times \Sigma \longrightarrow C l(\mathfrak{g})$ which will permit us to give an explicit representation formula for a surface immersed in a 3-dimensional Lorentzian Lie group.

Let us consider the involutive anti-automorphism $\tau: C l(\mathfrak{g}) \longrightarrow C l(\mathfrak{g})$ defined as the linear extension of the transposition of vectors $x_{1} \cdots x_{k} \longmapsto x_{k} \cdots x_{1}$ and the map

$$
\begin{aligned}
\langle\langle\cdot, \cdot\rangle\rangle: \quad C l(\mathfrak{g}) \times C l(\mathfrak{g}) & \longrightarrow C l(\mathfrak{g}) \\
\left(\sigma_{1}, \sigma_{2}\right) & \longmapsto \tau\left(\sigma_{2}\right) \sigma_{1} .
\end{aligned}
$$

If $g \in \operatorname{Spin}(\mathfrak{g})$ and $v, w \in C l(\mathfrak{g})$ we have

$$
\langle\langle g \cdot v, g \cdot w\rangle\rangle=\tau(g \cdot w) \cdot g \cdot v=\tau(w) \cdot \tau(g) \cdot g \cdot v=\tau(w) \cdot v=\langle\langle v, w\rangle\rangle,
$$

which shows that $\langle\langle\cdot, \cdot\rangle\rangle$ is $\operatorname{Spin}(\mathfrak{g})$-equivariant and induces a map

$$
\begin{array}{rlll}
\langle\langle\cdot, \cdot\rangle\rangle: \quad \Sigma \times \Sigma & \longrightarrow C l(\mathfrak{g}) \\
(\varphi, \psi) & \longmapsto \tau([\psi])[\varphi],
\end{array}
$$

where the brackets $[\varphi],[\psi] \in C l(\mathfrak{g})$ are the coordinates of $\varphi, \psi$ in a spinorial frame. The proofs of following two lemmas can be found in [4].

Lemma 2.1. For all $\varphi, \psi \in \Gamma(\Sigma)$ and $X \in \Gamma(T M \oplus E)$

$$
\langle\langle\varphi, \psi\rangle\rangle=\tau\langle\langle\psi, \varphi\rangle\rangle
$$

and

$$
\langle\langle X \cdot \varphi, \psi\rangle\rangle=\langle\langle\varphi, X \cdot \psi\rangle\rangle .
$$

Lemma 2.2. The connection $\nabla$ is compatible with the product $\langle\langle\cdot, \cdot\rangle\rangle$, that is,

$$
\partial_{X}\langle\langle\varphi, \psi\rangle\rangle=\left\langle\left\langle\nabla_{X} \varphi, \psi\right\rangle\right\rangle+\left\langle\left\langle\varphi, \nabla_{X} \psi\right\rangle\right\rangle
$$

for all $\varphi, \psi \in \Gamma(\Sigma)$ and $X \in T M$.

\subsection{Representation of a surface in a 3-dimensional Lorentzian Lie group.}

Let $G$ be a 3-dimensional Lorentzian Lie group endowed with a left-invariant metric $\langle\cdot, \cdot\rangle$ and $(M, g)$ an oriented pseudo-Riemmanian surface of signature $\left(r_{1}, s_{1}\right)$. We consider the trivial bundle $E=M \times \mathbb{R}$ with metric $-d \nu^{2}$ if $M$ is Riemannian or $+d \nu^{2}$ if $M$ is Lorentzian, and suppose that a symmetric bilinear form $B: T M \times T M \longrightarrow E$ is given. The following compatibility conditions on $M, G$ and $B$ will appear to be necessary to state our theorem.

(1) There exists a bundle isomorphism

$$
f: T M \oplus E \longrightarrow M \times \mathfrak{g}
$$

which preserves the metrics; from this isomorphism we define

$$
\Gamma: T M \oplus E \longrightarrow \Lambda^{2}(T M \oplus E) \subset C l_{\Sigma}
$$


such that, for all $X, Y \in \Gamma(T M \oplus E)$,

$$
f(\Gamma(X)(Y))=\Gamma(f(X))(f(Y))
$$

where in the right hand side of the equation, $\Gamma$ is the application defined in (2). Furthermore, we say that a section $Z$ of $T M \oplus E$ is left-invariant if $f(Z): M \longrightarrow \mathfrak{g}$ is a constant map.

(2) If $\nabla:=\nabla^{M} \oplus \nabla^{E}$ is the sum of the Levi-Civita connection on $M$ and the trivial connection on $E$, we assume that the covariant derivative of a left-invariant section $Z \in \Gamma(T M \oplus E)$ is

$$
\nabla_{X} Z=\Gamma(X)(Z)-B\left(X, Z^{T}\right)+B^{*}\left(X, Z^{N}\right)
$$

for all $X \in T M$, where $Z=Z^{T}+Z^{N} \in T M \oplus E$ and $B^{*}: T M \times E \longrightarrow T M$ is the bilinear operator that satisfies

$$
\langle B(X, Y), N\rangle=\left\langle Y, B^{*}(X, N)\right\rangle,
$$

for all $X, Y \in \Gamma(T M)$ and $N \in \Gamma(E)$.

Now, we enunciate the main theorem of that section whose proof in a slightly different context can be found in [4].

Theorem 1. We suppose that $M$ is simply connected. The following statements are equivalent:

(1) There exists a section $\varphi \in \Gamma(U \Sigma)$ such that

$$
\nabla_{X} \varphi=-\frac{1}{2} \sum_{j=1}^{2} \varepsilon_{j} e_{j} \cdot B\left(X, e_{j}\right) \cdot \varphi+\frac{1}{2} \Gamma(X) \cdot \varphi, \quad \varepsilon_{j}=g\left(e_{j}, e_{j}\right)
$$

for all $X \in T M$.

(2) There exists an isometric immersion $F: M \rightarrow G$ with normal bundle $E$ and second fundamental form $B$.

Precisely, if $\varphi$ is a solution of equation (13), replacing $\varphi$ by $\varphi \cdot$ a for some $a \in$ $\operatorname{Spin}(\mathfrak{g})$ if necessary, we consider the $\mathfrak{g}$-valued 1-form given by

$$
\xi(X)=\langle\langle X \cdot \varphi, \varphi\rangle\rangle,
$$

for all $X \in T M$ and the formula $F=\int \xi$ defines an isometric immersion $F$ : $M \longrightarrow G$ with normal bundle $E$ and second fundamental form $B$. Here $\int$ denotes the Darboux integral (see [17, Pag. 165]), i.e., $F=\int \xi: M \longrightarrow G$ is such that $F^{*} \omega=\xi$ for $\omega \in \Omega^{1}(G, \mathfrak{g})$ the Maurer-Cartan form defined in (1). Reciprocally, an isometric immersion $M \longrightarrow G$ with normal bundle $E$ and second fundamental form $B$ can be written in that way.

The explicit representation formula $F=\int \xi$ is a generalized Weierstrass formula for Lie groups.

Remark 2. Let us write the compatibility conditions using a frame $\left(e_{1}^{o}, e_{2}^{o}, e_{3}^{o}\right)$ of $\mathfrak{g}$ such that $\left\langle e_{i}^{o}, e_{j}^{o}\right\rangle=0$ if $i \neq j$ and $\left\langle e_{1}^{o}, e_{1}^{o}\right\rangle=\left\langle e_{2}^{o}, e_{2}^{o}\right\rangle=1=-\left\langle e_{3}^{o}, e_{3}^{o}\right\rangle$. We denote by $\Gamma_{i j}^{k} \in \mathbb{R}, 1 \leq i, j, k \leq 3$ the constants that satisfy

$$
\Gamma\left(e_{i}^{o}\right)\left(e_{j}^{o}\right)=\sum_{k=1}^{3} \varepsilon_{k} \Gamma_{i j}^{k} e_{k}^{o}, \quad \varepsilon_{k}=\left\langle e_{k}^{o}, e_{k}^{o}\right\rangle= \pm 1,
$$

and let $N$ be a unit section of $E:\langle N, N\rangle=\varepsilon$, where $\varepsilon=-1$ if $M$ is a Riemannian surface and $\varepsilon=+1$ if $M$ is a Lorentzian surface. For $i \in\{1,2,3\}$ we choose 
$\underline{e}_{i} \in \Gamma(T M \oplus E)$ such that $f\left(\underline{e}_{i}\right)=e_{i}^{o}$, and consider $\nu_{i} \in C^{\infty}(M)$ and $T_{i} \in \Gamma(T M)$ so that $\underline{e}_{i}=T_{i}+\nu_{i} N$; since $f$ is an isometry $\left(\underline{e}_{1}, \underline{e}_{2}, \underline{e}_{3}\right)$ is an orthonormal frame of $T M \oplus E$ and we have

$$
\left\langle T_{i}, T_{j}\right\rangle+\varepsilon \nu_{i} \nu_{j}=\varepsilon_{i} \delta_{i j},
$$

for all $i, j \in\{1,2,3\}$. With this notation, the equation (12) is equivalent to

$$
\begin{aligned}
\nabla_{X} T_{j} & =\sum_{1 \leq i, k \leq 3} \varepsilon_{i} \varepsilon_{k} \Gamma_{i j}^{k}\left\langle X, T_{i}\right\rangle T_{k}+\nu_{j} S(X) \\
d \nu_{j}(X) & =\sum_{1 \leq i, k \leq 3} \varepsilon_{i} \varepsilon_{k} \Gamma_{i j}^{k}\left\langle X, T_{i}\right\rangle \nu_{k}-h\left(X, T_{j}\right), \quad 1 \leq j \leq 3,
\end{aligned}
$$

where $S(X):=B^{*}(X, N)$ and $h\left(X, T_{j}\right):=\left\langle B\left(X, T_{j}\right), N\right\rangle$. Reciprocally, if there exist vector fields $T_{i} \in \Gamma(T M)$ and functions $\nu_{i} \in C^{\infty}(M)$ for $i \in\{1,2,3\}$ such that (15)-(17) are satisfied, then $f: T M \oplus E \longrightarrow M \times \mathfrak{g}$ given by $f\left(\underline{e}_{i}\right)=e_{i}^{o}$ for $\underline{e}_{i}=T_{i}+\nu_{i} N$ is a bundle isomorphism that preserves the metrics and such that (12) is satisfied.

\section{INTRINSIC VERSION OF THE IMMERSION THEOREM IN LIE GROUPS}

In this section we rewrite Theorem 1 using only the usual spinor bundle $\Sigma M$.

3.1. Immersion of a Riemannian surface. Let $M$ be a simply connected Riemannian surface and let $E=M \times \mathbb{R}$ be endowed with the metric $-d \nu^{2}$ in each fiber. For a symmetric bilinear form $B: T M \times T M \longrightarrow E$ and a section $N$ of $E$ such that $\langle N, N\rangle=-1$, we define the symmetric operator $S: T M \longrightarrow T M$ so that $\langle B(X, Y), N\rangle=\langle S(X), Y\rangle$ for all $X, Y \in T M$; explicitly, for all $X \in T M$

$$
S(X)=\sum_{j} e_{j}\left\langle B\left(X, e_{j}\right), N\right\rangle .
$$

We assume that there exist vector fields $T_{i} \in \Gamma(T M)$ and functions $\nu_{i} \in C^{\infty}(M)$, $i=1,2,3$, satisfying the compatibility conditions (15)-(17). Let us set for all $X \in T M$

$$
\Gamma_{1}(X)=\sum_{i=1}^{3} \varepsilon_{i}\left\langle X, T_{i}\right\rangle \sum_{j<k} \varepsilon_{j} \varepsilon_{k} \Gamma_{i j}^{k} \frac{1}{2}\left(T_{j} \cdot T_{k}-T_{k} \cdot T_{j}\right)
$$

and

$$
\Gamma_{2}(X)=\sum_{i=1}^{3} \varepsilon_{i}\left\langle X, T_{i}\right\rangle \sum_{j<k} \varepsilon_{j} \varepsilon_{k} \Gamma_{i j}^{k}\left(\nu_{j} T_{k}-\nu_{k} T_{j}\right) .
$$

The decomposition $C l_{1,2}=C l_{1,2}^{0} \oplus C l_{1,2}^{1}$ induces a splitting $\Sigma=\Sigma_{0} \oplus \Sigma_{1}$ of the spinor bundle, and there is a natural identification

$$
\begin{aligned}
\Sigma M & \rightarrow \Sigma_{0} \\
\psi & \mapsto \psi^{*}
\end{aligned}
$$

such that

$$
\left(\nabla_{X} \psi\right)^{*}=\nabla_{X} \psi^{*}, \quad(X \cdot \psi)^{*}=i N \cdot X \cdot \psi^{*}, \quad\left|\psi^{+}\right|^{2}-\left|\psi^{-}\right|^{2}=\left\langle\left\langle\psi^{*}, \psi^{*}\right\rangle\right\rangle
$$

for all $X \in T M$ (details are given in Appendix A).

Theorem 2. The following statements are equivalent:

(1) There exists an isometric immersion of $M$ into $G$ with shape operator $S$. 
(2) There exists $\psi \in \Gamma(\Sigma M)$ solution of

$$
\nabla_{X} \psi=\frac{i}{2} S(X) \cdot \psi+\frac{1}{2} \Gamma_{1}(X) \cdot \psi+\frac{i}{2} \Gamma_{2}(X) \cdot \psi
$$

for all $X \in T M$ and such that $\left|\psi^{+}\right|^{2}-\left|\psi^{-}\right|^{2}=1$.

The proof of theorem comes after the following lemmas.

Recall the notation of Remark 2: $\left(\underline{e}_{1}, \underline{e}_{2}, \underline{e}_{3}\right)$ is an orthonormal basis of invariant vector fields in $T M \oplus E$, and for $k=1,2,3, T_{k} \in \Gamma(T M)$ and $\nu_{k} \in C^{\infty}(M)$ are such that $\underline{e}_{k}=T_{k}+\nu_{k} N$. The proof of the following lemma can be found in a different context in [4].

Lemma 3.1. For all $X \in T M$,

$$
\Gamma(X)=\sum_{i} \varepsilon_{i}\left\langle X, T_{i}\right\rangle \sum_{j<k} \varepsilon_{j} \varepsilon_{k} \Gamma_{i j}^{k}\left(\frac{1}{2}\left(T_{j} \cdot T_{k}-T_{k} \cdot T_{j}\right)+\left(\nu_{k} T_{j}-\nu_{j} T_{k}\right) \cdot N\right),
$$

where $\varepsilon_{i}=\left\langle\underline{e}_{i}, \underline{e}_{i}\right\rangle= \pm 1$.

After some calculations we have from the properties in (19) the following lemma (details can be found in [28]).

Lemma 3.2. For $T_{j}, T_{k} \in \Gamma(T M), \nu_{j}, \nu_{k} \in C^{\infty}(M), N$ a unit section of $E$ and $\psi$ a spinor field of $\Sigma M$ the following identities are true:

$$
\left(T_{j} \cdot T_{k}-T_{k} \cdot T_{j}\right) \cdot \psi^{*}=\left(\left(T_{j} \cdot T_{k}-T_{k} \cdot T_{j}\right) \cdot \psi\right)^{*},
$$

$$
\left(\nu_{k} T_{j}-\nu_{j} T_{k}\right) \cdot N \cdot \psi^{*}=\left(i\left(\nu_{k} T_{j}-\nu_{j} T_{k}\right) \cdot \psi\right)^{*},
$$

$$
-\frac{1}{2} \sum_{j=1}^{2} e_{j} \cdot B\left(X, e_{j}\right) \cdot \psi^{*}=\left(\frac{i}{2} S(X) \cdot \psi\right)^{*} .
$$

Proof of Theorem 2. Let $\psi \in \Gamma(\Sigma M)$ be a solution of (20) such that $\left|\psi^{+}\right|^{2}-\left|\psi^{-}\right|^{2}=$ 1. If we apply the isomorphism (18) to both sides of that equation we deduce from Lemmas 3.1 and 3.2 that

$$
\left(\nabla_{X} \psi\right)^{*}=\left(\frac{i}{2} S(X) \cdot \psi\right)^{*}+\left(\frac{1}{2} \Gamma_{1}(X) \cdot \psi+\frac{i}{2} \Gamma_{2}(X) \cdot \psi\right)^{*}
$$

is equivalent to

$$
\nabla_{X} \psi^{*}=-\frac{1}{2} \sum_{j=1}^{2} e_{j} \cdot B\left(X, e_{j}\right) \cdot \psi^{*}+\frac{1}{2} \Gamma(X) \cdot \psi^{*},
$$

where $\varphi:=\psi^{*}$ is in $\Gamma(U \Sigma)$; Equation (22) is equivalent to (13) and the conclusion follows from Theorem 1 .

The following corollary gives a new proof of the spinorial characterization of the immersions in $\mathbb{R}^{1,2}$ obtained in [3]. See [14] for a result using two spinor fields.

Corollary 1. If $G=\mathbb{R}_{1}^{3}$ the following statements are equivalent:

(1) There exists an isometric immersion of $M$ into $\mathbb{R}_{1}^{3}$ with shape operator $S$. 
(2) There exists $\psi \in \Gamma(\Sigma M)$ solution of

$$
\nabla_{X} \psi=\frac{i}{2} S(X) \cdot \psi
$$

for all $X \in T M$ and such that $\left|\psi^{+}\right|^{2}-\left|\psi^{-}\right|^{2}=1$.

Proof. In this case $\Gamma_{i j}^{k}=0$ for all $i, j, k \in\{1,2,3\}$ and then $\Gamma_{1}(X)=0=\Gamma_{2}(X)$

Remark 3. If $\psi=\psi^{+}+\psi^{-}$is as in Theorem 2 then the corresponding 1-form $\xi(X)=\langle\langle X \cdot \varphi, \varphi\rangle\rangle$ of Theorem 1 reads

$$
\xi(X)=2 i I \Im m\left\langle X \cdot \psi^{-}, \psi^{+}\right\rangle+J\left(\left\langle X \cdot \psi^{+}, \alpha\left(\psi^{+}\right)\right\rangle-\left\langle X \cdot \psi^{-}, \alpha\left(\psi^{-}\right)\right\rangle\right),
$$

where $\alpha: \Sigma M \longrightarrow \Sigma M$ is a quaternionic structure; the immersion is thus explicitly given in terms of $\psi$; the proof may be found in [28, Proposition 3.4.13].

3.2. Immersion of a Lorentzian surface. Let $(M, g)$ be a simply connected Lorentzian surface and let $E=M \times \mathbb{R}$ be endowed with the metric $+d \nu^{2}$ in each fiber. Let $B: T M \times T M \longrightarrow E$ be a bilinear symmetric form and consider its associated symmetric operator $S: T M \longrightarrow T M$ given by

$$
S(X)=\sum_{j=1}^{2} \varepsilon_{j}\left\langle B\left(X, e_{j}\right), N\right\rangle e_{j}, \varepsilon_{j}=\left\langle e_{j}, e_{j}\right\rangle= \pm 1
$$

for all $X \in T M$ and $N$ a fixed unit section of $E$. We assume that there exist vector fields $T_{i} \in \Gamma(T M)$ and functions $\nu_{i} \in C^{\infty}(M), i=1,2,3$, satisfying the compatibility conditions (15)-(17). Let us set for all $X \in T M$

$$
\tilde{\Gamma}(X)=\sum_{i} \varepsilon_{i}\left\langle X, T_{i}\right\rangle \sum_{j<k} \varepsilon_{j} \varepsilon_{k} \Gamma_{i j}^{k}\left(\frac{1}{2}\left(T_{j} \cdot T_{k}-T_{k} \cdot T_{j}\right)+\left(\nu_{k} T_{j}-, \nu_{j} T_{k}\right)\right) .
$$

The decomposition $C l_{1,2}=C l_{1,2}^{0} \oplus C l_{1,2}^{1}$ induces a splitting $\Sigma=\Sigma_{0} \oplus \Sigma_{1}$ of the spinor bundle, and there is a natural identification

$$
\begin{aligned}
\Sigma M & \rightarrow \Sigma_{0} \\
\psi & \mapsto \psi^{*}
\end{aligned}
$$

such that

$$
\left(\nabla_{X} \psi\right)^{*}=\nabla_{X} \psi^{*}, \quad(X \cdot \psi)^{*}=X \cdot N \cdot \psi^{*}, \quad\left|\psi^{+}\right|^{2}-\left|\psi^{-}\right|^{2}=\left\langle\left\langle\psi^{*}, \psi^{*}\right\rangle\right\rangle
$$

for all $X \in T M$ (details are given in Appendix A).

Theorem 3. The following statements are equivalent:

(1) There exists an isometric immersion of $M$ into $G$ with shape operator $S$.

(2) There exists $\psi \in \Gamma(\Sigma M)$ solution of

$$
\nabla_{X} \psi=-\frac{1}{2} S(X) \cdot \psi+\frac{1}{2} \tilde{\Gamma}(X) \cdot \psi,
$$

for all $X \in T M$ and such that $\left|\psi^{+}\right|^{2}-\left|\psi^{-}\right|^{2}=1$.

Proof. Let $\psi$ be a section of $\Sigma M$ with $\left|\psi^{+}\right|^{2}-\left|\psi^{-}\right|^{2}=1$ that satisfies (28). If we apply the isomorphism (26) to that equation it follows from the properties (27) 
that $\left(\nabla_{X} \psi\right)^{*}=\nabla_{X} \psi^{*},\left(-\frac{1}{2} S(X) \cdot \psi\right)^{*}=-\frac{1}{2} \sum_{j=1}^{2} \varepsilon_{j} e_{j} \cdot B\left(X, e_{j}\right) \cdot \psi^{*}$, and, if $\Gamma(X)$ and $\tilde{\Gamma}(X)$ are given by Lemma 3.1 and equation (25) respectively, that

$$
\left(\frac{1}{2} \tilde{\Gamma}(X) \cdot \psi\right)^{*}=\frac{1}{2} \Gamma(X) \cdot \psi^{*} .
$$

Therefore, the following equation

$$
\left(\nabla_{X} \psi\right)^{*}=\left(-\frac{1}{2} S(X) \cdot \psi\right)^{*}+\left(\frac{1}{2} \tilde{\Gamma}(X) \cdot \psi\right)^{*},
$$

is equivalent to

$$
\nabla_{X} \psi^{*}=-\frac{1}{2} \sum_{j=1}^{2} \varepsilon_{j} e_{j} \cdot B\left(X, e_{j}\right) \cdot \psi^{*}+\frac{1}{2} \Gamma(X) \cdot \psi^{*},
$$

so if we set $\varphi:=\psi^{*}$ the Equation (30) is equivalent to (13) and the result is a consequence of Theorem 1.

Corollary 2. If $M$ is a simply connected Lorentzian surface and $G=\mathbb{R}_{1}^{3}$ then the following statements are equivalent:

(1) There exists an isometric immersion of $M$ in $\mathbb{R}_{1}^{3}$ with shape operator $S$.

(2) There exists $\psi \in \Gamma(\Sigma M)$ solution of

$$
\nabla_{X} \psi=-\frac{1}{2} S(X) \cdot \psi
$$

for all $X \in T M$ and such that $\left|\psi^{+}\right|^{2}-\left|\psi^{-}\right|^{2}=1$.

See [12] and [14] for a similar result involving two spinor fields.

Proof. In that case $\Gamma_{i j}^{k}=0$ for all $i, j, k \in\{1,2,3\}$ and then $\tilde{\Gamma}(X)=0$ for all $X \in T M$.

\section{Representation of a SURFACE in LoREntzian PRODUCtS}

In this section we characterize the immersions of an orientable Riemannian surface $(M, g)$ in the symmetric Lorentzian products $\mathbb{H}^{2} \times \mathbb{R}_{-}, \mathbb{R} \times \mathbb{S}_{1}^{2}$ and $\mathbb{R} \times \mathbb{H}_{1}^{2}$ which are locally isometric to a Lie group with one of the following Lie algebras:

$$
\begin{aligned}
& {\left[e_{1}, e_{2}\right]=\alpha e_{2} \quad\left[e_{1}, e_{2}\right]=0 \quad\left[e_{1}, e_{2}\right]=0} \\
& \mathfrak{a}:\left[e_{1}, e_{3}\right]=0 \quad \mathfrak{b}:\left[e_{1}, e_{3}\right]=\alpha e_{1} \quad \mathfrak{c}:\left[e_{1}, e_{3}\right]=\delta e_{3} \\
& {\left[e_{2}, e_{3}\right]=0 \quad\left[e_{2}, e_{3}\right]=0 \quad\left[e_{2}, e_{3}\right]=0}
\end{aligned}
$$

where $\alpha, \delta \neq 0,\left\langle e_{1}, e_{1}\right\rangle=\left\langle e_{2}, e_{2}\right\rangle=1=-\left\langle e_{3}, e_{3}\right\rangle$ and $\left\langle e_{i}, e_{j}\right\rangle=0$ for $i \neq j$. By the Koszul formula, the only non-zero components for the covariant derivative $\bar{\nabla}$ are, in each case,

$$
\mathfrak{a}: \begin{aligned}
& \bar{\nabla}_{e_{2}} e_{1}=-\alpha e_{2} \\
& \bar{\nabla}_{e_{2}} e_{2}=\alpha e_{1},
\end{aligned} \quad \mathfrak{b}: \begin{aligned}
& \bar{\nabla}_{e_{1}} e_{1}=\alpha e_{3} \\
& \bar{\nabla}_{e_{1}} e_{3}=\alpha e_{1},
\end{aligned} \quad \mathfrak{c}: \begin{aligned}
& \bar{\nabla}_{e_{3}} e_{1}=-\delta e_{3} \\
& \bar{\nabla}_{e_{3}} e_{3}=-\delta e_{1} .
\end{aligned}
$$

We assume the following compatibility conditions (see Remark 2): there exist tangent vector fields $T_{i} \in \Gamma(T M)$ and functions $\nu_{i} \in C^{\infty}(M)$ such that

$$
\left\langle T_{i}, T_{j}\right\rangle-\nu_{i} \nu_{j}=\varepsilon_{i} \delta_{i j}, \quad 1 \leq i, j \leq 3
$$

and, for $1 \leq j \leq 3$,

$$
\nabla_{X} T_{j}=\sum_{1 \leq i, k \leq 3} \varepsilon_{i} \varepsilon_{k} \Gamma_{i j}^{k}\left\langle X, T_{i}\right\rangle T_{k}+\nu_{j} S(X)
$$


and

$$
d \nu_{j}(X)=\sum_{1 \leq i, k \leq 3} \varepsilon_{i} \varepsilon_{k} \Gamma_{i j}^{k}\left\langle X, T_{i}\right\rangle \nu_{k}-h\left(X, T_{j}\right)
$$

where $S(X)=B^{*}(X, N), h\left(X, T_{j}\right)=\left\langle B\left(X, T_{j}\right), N\right\rangle$ and $\varepsilon_{j}= \pm 1$.

Theorem 4. If $M$ is simply connected then the following statements are equivalent:

(1) There exists $\psi \in \Gamma(\Sigma M)$ solution of

$$
\nabla_{X} \psi=\frac{i}{2} S(X) \cdot \psi-\frac{\alpha}{2}\left\langle X, T_{2}\right\rangle\left(i T_{3}+\nu_{3}\right) \cdot \omega \cdot \psi
$$

for all $X \in T M$ and such that $\left|\psi^{+}\right|^{2}-\left|\psi^{-}\right|^{2}=1$. Here $\omega=\epsilon_{1} \cdot \epsilon_{2}$ where $\left(\epsilon_{1}, \epsilon_{2}\right)$ is a positively oriented orthonormal basis of $M$.

(2) There exists an isometric immersion of $M$ into $\mathbb{H}^{2} \times \mathbb{R}_{-}$with shape operator $S$.

Proof. We prove that the existence of a solution of equation (32) is equivalent to the existence of a solution of equation (13) and then use Theorem 1 to obtain the result. It follows from Lemma B.1 that the bivector representing $\Gamma(X)$ is

$$
\Gamma(X)=\frac{1}{2}\left(\underline{e}_{1} \cdot \Gamma(X)\left(\underline{e}_{1}\right)+\underline{e}_{2} \cdot \Gamma(X)\left(\underline{e}_{2}\right)-\underline{e}_{3} \cdot \Gamma(X)\left(\underline{e}_{3}\right)\right),
$$

where $\left(\underline{e}_{1}, \underline{e}_{2}, \underline{e}_{3}\right)$ is as in Remark 2. By (31) we have that

$$
\Gamma(X)\left(\underline{e}_{1}\right)=-\alpha\left\langle X, \underline{e}_{2}\right\rangle \underline{e}_{2}, \quad \Gamma(X)\left(\underline{e}_{2}\right)=\alpha\left\langle X, \underline{e}_{2}\right\rangle \underline{e}_{1} \quad \text { and } \quad \Gamma(X)\left(\underline{e}_{3}\right)=0,
$$

and then $\Gamma(X)=-\alpha\left\langle X, \underline{e}_{2}\right\rangle \underline{e}_{1} \cdot \underline{e}_{2}$. Since $\underline{e}_{k}=T_{k}+\nu_{k} N$ and $\underline{e}_{1} \cdot \underline{e}_{2} \cdot \underline{e}_{3}=\omega \cdot N$ it follows that

$$
\begin{aligned}
\Gamma(X) & =-\alpha\left\langle X, \underline{e}_{2}\right\rangle \underline{e}_{1} \cdot \underline{e}_{2} \\
& =-\alpha\left\langle X, \underline{e}_{2}\right\rangle \underline{e}_{1} \cdot \underline{e}_{2} \cdot \underline{e}_{3} \cdot \underline{e}_{3} \\
& =-\alpha\left\langle X, T_{2}\right\rangle \omega \cdot N \cdot\left(T_{3}+\nu_{3} N\right) \\
& =-\alpha\left\langle X, T_{2}\right\rangle\left(-N \cdot T_{3}+\nu_{3}\right) \cdot \omega .
\end{aligned}
$$

We now apply to the equation (32) the isomorphism (18) to obtain

$$
-\frac{1}{2} \sum_{j=1}^{2} e_{j} \cdot B\left(X, e_{j}\right) \cdot \psi^{*}=\left(\frac{i}{2} S(X) \cdot \psi\right)^{*}
$$

and

$$
\frac{1}{2} \Gamma(X) \cdot \psi^{*}=\left(-\frac{1}{2} \alpha\left\langle X, T_{2}\right\rangle\left(i T_{3}+\nu_{3}\right) \cdot \omega \cdot \psi\right)^{*} .
$$

Therefore, if $\varphi:=\psi^{*}$, the equation

$$
\nabla_{X} \varphi=-\frac{1}{2} \sum_{j=1}^{2} e_{j} \cdot B\left(X, e_{j}\right) \cdot \varphi+\frac{1}{2} \Gamma(X) \cdot \varphi
$$

is equivalent to (32).

The proofs of Theorems 5 and 6 below are similar to the proof of Theorem 4 so we omit them.

Theorem 5. If $M$ is simply connected, the following statements are equivalent: 
(1) There exists $\psi \in \Gamma(\Sigma M)$ solution of

$$
\nabla_{X} \psi=\frac{i}{2} S(X) \cdot \psi+\frac{\alpha}{2}\left\langle X, T_{1}\right\rangle\left(i T_{2}+\nu_{2}\right) \cdot \omega \cdot \psi
$$

for all $X \in T M$ and such that $\left|\psi^{+}\right|^{2}-\left|\psi^{-}\right|^{2}=1$.

(2) There exists an isometric immersion of $M$ into $\mathbb{R} \times \mathbb{S}_{1}^{2}$ with shape operator $S$.

Theorem 6. If $M$ is simply connected then the following statements are equivalent:

(1) There exists $\psi \in \Gamma(\Sigma M)$ solution of

$$
\nabla_{X} \psi=\frac{i}{2} S(X) \cdot \psi+\frac{\alpha}{2}\left\langle X, T_{3}\right\rangle\left(i T_{2}+\nu_{2}\right) \cdot \omega \cdot \psi
$$

for all $X \in T M$ and such that $\left|\psi^{+}\right|^{2}-\left|\psi^{-}\right|^{2}=1$.

(2) There exists an isometric immersion of $M$ into $\mathbb{R} \times \mathbb{H}_{1}^{2}$ with shape operator $S$.

\section{Representation of A SURFACE IN $\mathbb{S}_{1}^{3}$ AND $\mathbb{H}_{1}^{3}$}

5.1. Representation of a surface in $\mathbb{S}_{1}^{3}$. The 3-dimensional de Sitter space is the Lorentzian hypersurface of $\mathbb{R}^{1,3}$ with constant sectional curvature 1 given by the quadric

$$
\mathbb{S}_{1}^{3}=\left\{\left(x_{1}, x_{2}, x_{3}, x_{4}\right) \in \mathbb{R}^{1,3}:-x_{1}^{2}+x_{2}^{2}+x_{3}^{2}+x_{4}^{2}=1\right\} .
$$

We suppose that $M$ is a simply connected Riemannian surface and $E=M \times \mathbb{R}$ is the trivial bundle with metric $-d \nu^{2}$ in the fibers. Let us assume that a symmetric bilinear form $B: T M \times T M \longrightarrow E$ is given.

We consider the splitting $\mathbb{R}^{1,3}=\mathbb{R}^{1,2} \oplus \mathbb{R} e_{4}$ where $e_{4}$ is the last element of the standard orthonormal basis of $\mathbb{R}^{1,3}$, the corresponding application

$$
\begin{aligned}
\operatorname{Spin}(0,2) \times \operatorname{Spin}(1,0) & \longrightarrow \operatorname{Spin}(1,2) \subset \operatorname{Spin}(1,3) \\
\left(g_{1}, g_{2}\right) & \longmapsto g_{1} g_{2},
\end{aligned}
$$

and the representation

$$
\begin{aligned}
\rho: \operatorname{Spin}(0,2) \times \operatorname{Spin}(1,0) & \longrightarrow G l\left(C l_{1,3}\right) \\
\left(g_{1}, g_{2}\right) & \longmapsto \rho\left(g_{1}, g_{2}\right): C l_{1,3} \quad \longrightarrow \quad C l_{1,3}
\end{aligned}
$$

For $\widetilde{Q}=\widetilde{Q}_{M} \times_{M} \widetilde{Q}_{E}$ the product of spin structures, we define the bundles

$$
\Sigma:=\widetilde{Q} \times{ }_{\rho} C l_{1,3}, \quad U \Sigma:=\widetilde{Q} \times_{\rho} \operatorname{Spin}(1,3) \subset \Sigma \text { and } C l_{\Sigma}:=\widetilde{Q} \times_{A d} C l_{1,3},
$$

and the element $\nu=\left[\widetilde{s}, e_{4}\right] \in C l_{\Sigma}$ (it is well-defined, independently of the choice of $\tilde{s} \in \widetilde{Q}$, since by construction $e_{4}$ is invariant by the action of $\operatorname{Spin}(1,2)$ ).

Theorem 7. The following statements are equivalent:

(1) There exists $\varphi \in \Gamma(U \Sigma)$ solution of

$$
\nabla_{X} \varphi=-\frac{1}{2} \sum_{j=1}^{2} e_{j} \cdot B\left(X, e_{j}\right) \cdot \varphi+\frac{1}{2} X \cdot \nu \cdot \varphi
$$

for all $X \in T M$. 
SPINORIAL REPRESENTATION OF SURFACES IN LORENTZIAN HOMOGENEOUS SPACES OF DIMENSION13

(2) There exists an isometric immersion $F$ of $M$ into $\mathbb{S}_{1}^{3}$ with second fundamental form $B$.

Moreover, $F: M \longrightarrow \mathbb{S}_{1}^{3}$ is given by

$$
F=\langle\langle\nu \cdot \varphi, \varphi\rangle\rangle .
$$

Proof. We first prove $1 \Longrightarrow 2$. Let us suppose that $\varphi \in \Gamma(U \Sigma)$ is a solution of (36) and observe that $F$ defined by (37) takes values in $\mathbb{S}_{1}^{3}$ : by definition, $F=\tau[\varphi][\nu][\varphi]$ and since $[\varphi] \in \operatorname{Spin}(1,3)$ and $[\nu] \in \mathbb{R}^{1,3}$ we have that $F=\operatorname{Ad}\left([\varphi]^{-1}\right)([\nu])$. Since $\operatorname{Ad}\left([\varphi]^{-1}\right) \in S O(1,3)$ and $[\nu]$ has norm 1 we conclude that $\operatorname{Ad}\left([\varphi]^{-1}\right)([\nu]) \in \mathbb{S}_{1}^{3}$. The rest of the proof rely on the following results, the proof of the first one can be found in [2, Lemma 8.1] and we omit it here:

Lemma 5.1. If $\varphi \in \Gamma(U \Sigma)$ satisfies equation (36) and $F: M \longrightarrow \mathbb{S}_{1}^{3}$ is defined by (37) then for all $X \in T M$

$$
d F(X)=\langle\langle X \cdot \varphi, \varphi\rangle\rangle .
$$

Proposition 5.2. The following statements are true:

(1) $F: M \longrightarrow \mathbb{S}_{1}^{3}$ is an isometry.

(2) The application

$$
\begin{aligned}
\Phi_{E}: E & \longrightarrow T \mathbb{S}_{1}^{3} \\
X \in E_{m} & \longmapsto(F(m),\langle\langle X \cdot \varphi, \varphi\rangle\rangle)
\end{aligned}
$$

is an isomorphism between $E$ and the normal bundle of the immersion $F$ which preserves the metrics and identifies $B$ with the second fundamental form of $F$.

Proof. For simplicity let us set $\xi(X):=\langle\langle X \cdot \varphi, \varphi\rangle\rangle$ for all $X \in T M \oplus E$, then, for all $X, Y \in T M \oplus E$

$$
\begin{aligned}
\langle\xi(X), \xi(Y)\rangle & =-\frac{1}{2}(\xi(X) \xi(Y)+\xi(Y) \xi(X)) \\
& =-\frac{1}{2}(\tau[\varphi][X][\varphi] \tau[\varphi][Y][\varphi]+\tau[\varphi][Y][\varphi] \tau[\varphi][X][\varphi]) \\
& =-\frac{1}{2} \tau[\varphi]([X][Y]+[Y][X])[\varphi] \\
& =\tau[\varphi]\langle X, Y\rangle[\varphi] \\
& =\langle X, Y\rangle,
\end{aligned}
$$

which proves that $F$ and $\Phi_{E}$ are isometries. Let us see now that $B$ is the second fundamental form of the immersion, in other words, if $N M$ is the normal bundle of $F$ and $B^{F}: T M \times T M \longrightarrow N M$ is its second fundamental, then

$$
B^{F}(X, Y)=\langle\langle B(X, Y) \cdot \varphi, \varphi\rangle\rangle .
$$

Let $X, Y$ be sections of $T M$ such that $\nabla X=0=\nabla Y$ at a point; by definition

$$
B^{F}(X, Y)=\left\{\partial_{X}(d F(Y))\right\}^{N}=\left(\partial_{X}\langle\langle Y \cdot \varphi, \varphi\rangle\rangle\right)^{N} .
$$


Since $\varphi \in \Gamma(U \Sigma)$ is a solution of (36) it follows that

$$
\begin{aligned}
\partial_{X}\langle\langle Y \cdot \varphi, \varphi\rangle\rangle= & \left\langle\left\langle Y \cdot \nabla_{X} \varphi, \varphi\right\rangle\right\rangle+\left\langle\left\langle Y \cdot \varphi, \nabla_{X} \varphi\right\rangle\right\rangle \\
= & (i d+\tau)\left\langle\left\langle Y \cdot \nabla_{X} \varphi, \varphi\right\rangle\right\rangle \\
= & -\frac{1}{2}(i d+\tau)\left\langle\left\langle Y \cdot \sum_{j=1}^{2} e_{j} \cdot B\left(X, e_{j}\right) \cdot \varphi, \varphi\right\rangle\right\rangle \\
& +\frac{1}{2}(i d+\tau)\langle\langle Y \cdot X \cdot \nu \cdot \varphi, \varphi\rangle\rangle .
\end{aligned}
$$

We first prove the following equality

$$
-\frac{1}{2}(i d+\tau)\left\langle\left\langle Y \cdot \sum_{j=1}^{2} e_{j} \cdot B\left(X, e_{j}\right) \cdot \varphi, \varphi\right\rangle\right\rangle=\langle\langle B(X, Y) \cdot \varphi, \varphi\rangle\rangle .
$$

If $X=\sum_{i=1}^{2} x_{i} e_{i}$ and $Y=\sum_{k=1}^{2} y_{k} e_{k}$, then

$$
\begin{aligned}
Y \cdot \sum_{j=1}^{2} e_{j} \cdot B\left(X, e_{j}\right) & =\sum_{k=1}^{2} \sum_{j=1}^{2} y_{k} e_{k} \cdot e_{j} \cdot B\left(X, e_{j}\right) \\
& =-\sum_{j} y_{j} B\left(X, e_{j}\right)+\sum_{k \neq j} y_{k} e_{k} \cdot e_{j} \cdot B\left(X, e_{j}\right) \\
& =-B(X, Y)+\sum_{k \neq j} y_{k} e_{k} \cdot e_{j} \cdot B\left(X, e_{j}\right),
\end{aligned}
$$

therefore,

$$
\begin{aligned}
\left\langle\left\langle Y \cdot \sum_{j=1}^{2} e_{j} \cdot B\left(X, e_{j}\right) \cdot \varphi, \varphi\right\rangle\right\rangle & =\langle\langle-B(X, Y) \cdot \varphi, \varphi\rangle\rangle \\
& +\sum_{k \neq j}\left\langle\left\langle y_{k} e_{k} \cdot e_{j} \cdot B\left(X, e_{j}\right) \cdot \varphi, \varphi\right\rangle\right\rangle
\end{aligned}
$$

and

$$
\begin{aligned}
\tau\left\langle\left\langle Y \cdot \sum_{j=1}^{2} e_{j} \cdot B\left(X, e_{j}\right) \cdot \varphi, \varphi\right\rangle\right\rangle & =\langle\langle-B(X, Y) \cdot \varphi, \varphi\rangle\rangle \\
& -\sum_{k \neq j}\left\langle\left\langle y_{k} e_{k} \cdot e_{j} \cdot B\left(X, e_{j}\right) \cdot \varphi, \varphi\right\rangle\right\rangle,
\end{aligned}
$$

which implies (39). Using (38) and (39), it is enough to finish the proof to verify that $\frac{1}{2}(i d+\tau)\langle\langle Y \cdot X \cdot \nu \cdot \varphi, \varphi\rangle\rangle$ does not have normal component. We have that

$$
\langle\langle Y \cdot X \cdot \nu \cdot \varphi, \varphi\rangle\rangle=\sum_{i \neq k} y_{i} x_{k} \tau[\varphi]\left[e_{i}\right] \cdot\left[e_{k}\right] \cdot[\nu] \cdot[\varphi]-\sum_{k} y_{k} x_{k} \tau[\varphi] \cdot[\nu][\varphi]
$$

and therefore

$$
\tau\langle\langle Y \cdot X \cdot \nu \cdot \varphi, \varphi\rangle\rangle=-\sum_{i \neq k} y_{i} x_{k} \tau[\varphi]\left[e_{i}\right] \cdot\left[e_{k}\right] \cdot[\nu] \cdot[\varphi]-\sum_{k} y_{k} x_{k} \tau[\varphi] \cdot[\nu][\varphi],
$$

which implies

$$
\left.\frac{1}{2}(i d+\tau)\langle\langle Y \cdot X \cdot \nu \cdot \varphi, \varphi)\rangle\right\rangle=-\sum_{k} y_{k} x_{k} \tau[\varphi] \cdot[\nu] \cdot[\varphi],
$$


which has no component normal to the immersion because for $N$ a section of $E$, $[N]$ and $[\nu]=e_{4}$ are orthogonal.

We finally prove $2 \Longrightarrow 1$. Suppose that $M$ is isometrically immersed in $\mathbb{S}_{1}^{3} \subset \mathbb{R}^{1,3}$ and consider the section $\left.\varphi \in \Sigma \mathbb{R}^{1,3}\right|_{M}$ given by $\varphi=\left[1_{\operatorname{Spin}(1,3)}, 1_{C l_{\mathbb{R}^{1,3}}}\right]$; it belongs to $U \Sigma$ and using the spinorial Gauss formula we deduce that it satisfies (36).

5.2. Representation of a surface in $\mathbb{H}_{1}^{3}$. The pseudo-hyperbolic space of dimension 3 is the Lorentzian hypersurface of $\mathbb{R}^{2,2}$ with constant sectional curvature -1 given by the quadric

$$
\mathbb{H}_{1}^{3}=\left\{\left(x_{1}, x_{2}, x_{3}, x_{4}\right) \in \mathbb{R}^{2,2}:-x_{1}^{2}-x_{2}^{2}+x_{3}^{2}+x_{4}^{2}=-1\right\} .
$$

We suppose that $M$ is a simply connected Riemannian surface and $E:=M \times \mathbb{R}$ is the trivial bundle with metric $-d \nu^{2}$ in the fibers. Let us assume that $B: T M \times T M \longrightarrow$ $E$ is a given symmetric bilinear form.

We consider the splitting $\mathbb{R}^{2,2}=\mathbb{R} e_{1} \oplus \mathbb{R}^{1,2}$ where $e_{1}$ is the first vector of the standard orthonormal basis of $\mathbb{R}^{2,2}$ so that $\left\langle e_{1}, e_{1}\right\rangle=-1$, the application

$$
\begin{aligned}
\operatorname{Spin}(0,2) \times \operatorname{Spin}(1,0) & \longrightarrow \operatorname{Spin}(1,2) \subset \operatorname{Spin}(2,2) \\
\left(g_{1}, g_{2}\right) & \longmapsto g_{1} g_{2}
\end{aligned}
$$

where the inclusion $\operatorname{Spin}(1,2) \subset \operatorname{Spin}(2,2)$ corresponds to the inclusion $\mathbb{R}^{1,2} \subset \mathbb{R}^{2,2}$ in the splitting above, and the representation

$$
\begin{aligned}
& \rho: \quad \operatorname{Spin}(0,2) \times \operatorname{Spin}(1,0) \longrightarrow G l\left(\mathrm{Cl}_{2,2}\right) \\
& \left(g_{1}, g_{2}\right) \longmapsto \rho\left(g_{1}, g_{2}\right): C l_{2,2} \quad \longrightarrow \quad C l_{2,2} \\
& v \longmapsto g_{1} g_{2} \cdot v .
\end{aligned}
$$

Denoting by $\widetilde{Q}$ a product of spin structures of $T M$ and $E$, we define the bundles

$$
\Sigma:=\widetilde{Q} \times_{\rho} C l_{2,2}, \quad U \Sigma:=\widetilde{Q} \times_{\rho} \operatorname{Spin}(2,2) \subset \Sigma \text { and } C l_{\Sigma}:=\widetilde{Q} \times_{A d} C l_{2,2}
$$

and set $\nu:=\left[\tilde{s}, e_{1}\right] \in C l_{\Sigma}$. The proof of the following theorem is similar to the proof of Theorem 7 and we omit it.

Theorem 8. The following statements are equivalent:

(1) There exists $\varphi \in \Gamma(U \Sigma)$ such that

$$
\nabla_{X} \varphi=-\frac{1}{2} \sum_{j=1}^{2} e_{j} \cdot B\left(X, e_{j}\right) \cdot \varphi-\frac{1}{2} X \cdot \nu \cdot \varphi
$$

for all $X \in T M$.

(2) There exists an isometric immersion $F$ of $M$ into $\mathbb{H}_{1}^{3}$ with second fundamental form $B$.

Moreover $F: M \longrightarrow \mathbb{H}_{1}^{3}$ is given by $F=\langle\langle\nu \cdot \varphi, \varphi\rangle\rangle$.

\section{Representation of A SURFACE IN $\mathbb{R}_{-} \times \mathbb{S}^{2}$}

The product manifold $\mathbb{R}_{-} \times \mathbb{S}^{2}$ is isometrically immersed in $\mathbb{R}^{1,3}$ as the following quadric

$$
\mathbb{R}_{-} \times \mathbb{S}^{2}=\left\{\left(t, x_{1}, x_{2}, x_{3}\right): x_{1}^{2}+x_{2}^{2}+x_{3}^{2}=1\right\} \subset \mathbb{R}^{1,3} .
$$


If $M$ is a surface isometrically immersed in $\mathbb{R}_{-} \times \mathbb{S}^{2} \subset \mathbb{R}^{1,3}$ then, by the Gauss formula, the restriction to $M$ of a constant spinor field $\varphi$ satisfies

$$
\nabla_{X} \varphi=-\frac{1}{2} \sum_{j=1}^{2} e_{j} \cdot B\left(X, e_{j}\right) \cdot \varphi+\frac{1}{2} X_{0} \cdot \nu \cdot \varphi,
$$

for all $X \in T M$, where $B$ is the second fundamental form of the immersion $M \hookrightarrow$ $\mathbb{R}_{-} \times \mathbb{S}^{2}, \nu$ is the outward normal vector field of $\mathbb{R}_{-} \times \mathbb{S}^{2}$ in $\mathbb{R}^{1,3}$ and $X_{0}$ is the projection of $X$ onto $T \mathbb{S}^{2}$. Suppose that $(M, g)$ is a simply connected Riemannian surface and $E=M \times \mathbb{R}$ is the trivial bundle with the metric $-d \nu^{2}$ in the fibers.

We consider the splitting $\mathbb{R}^{1,3}=\mathbb{R}^{1,2} \oplus \mathbb{R} e_{4}$, where $e_{4}$ is the last vector of the standard orthonormal basis of $\mathbb{R}^{1,3}$, the application

$$
\begin{aligned}
\operatorname{Spin}(0,2) \times \operatorname{Spin}(1,0) & \longrightarrow \operatorname{Spin}(1,2) \subset \operatorname{Spin}(1,3) \\
\left(g_{1}, g_{2}\right) & \longmapsto g_{1} g_{2}
\end{aligned}
$$

and the representation

$$
\begin{aligned}
\rho: \quad \operatorname{Spin}(0,2) \times \operatorname{Spin}(1,0) & \longrightarrow G l\left(C l_{1,3}\right) \\
\left(g_{1}, g_{2}\right) & \longmapsto \rho\left(g_{1}, g_{2}\right): C l_{1,3} \longrightarrow C_{1,3} \\
&
\end{aligned}
$$

If $\widetilde{Q}$ stands for a product of spin structures of $T M$ and $E$, we define the bundles

$$
\Sigma:=\tilde{Q} \times{ }_{\rho} C l_{1,3}, \quad U \Sigma:=\tilde{Q} \times_{\rho} \operatorname{Spin}(1,3) \subset \Sigma, \quad C l_{\Sigma}:=\tilde{Q} \times_{A d} C l_{1,3}
$$

and set $\nu:=\left[\widetilde{s}, e_{4}\right] \in C l_{\Sigma}$.

We consider a symmetric bilinear form $B: T M \times T M \longrightarrow E$ and denote by $S: T M \longrightarrow T M$ the symmetric operator such that $\langle S(X), Y\rangle=\langle B(X, Y), N\rangle$ for all $X, Y \in T M$ where $N$ is a given unit section of $E$. Let us suppose that there exist $T \in \Gamma(T M)$ and $f \in C^{\infty}(M)$ such that

$$
\begin{array}{r}
\|T\|^{2}-f^{2}=-1 \\
\nabla_{X} T=f S(X) \\
d f(X)=\langle S(X), T\rangle .
\end{array}
$$

It follows from (42) that $e_{0}:=T+f N$ has norm -1 .

Lemma 6.1. If $M$ is simply connected and $T \in \Gamma(T M)$ satisfies the equations (42)-(44) then there exists $\eta: M \longrightarrow \mathbb{R}$ such that $d \eta(X)=-\langle X, T\rangle$.

Proof. The latter holds since, for all $X, Y \in \Gamma(T M)$,

$$
\begin{aligned}
d \beta(X, Y) & =X . \beta(Y)-Y . \beta(X)-\beta([X, Y]) \\
& =\left\langle\nabla_{X} Y, T\right\rangle+\left\langle Y, \nabla_{X} T\right\rangle-\left\langle\nabla_{Y} X, T\right\rangle-\left\langle X, \nabla_{Y} T\right\rangle-\langle[X, Y], T\rangle \\
& =\langle Y, f S(X)\rangle-\langle X, f S(Y)\rangle \\
& =0 .
\end{aligned}
$$

Theorem 9. If $M$ is simply connected and $\eta: M \longrightarrow \mathbb{R}$ is the differentiable function of Lemma 6.1 then the following statements are equivalent: 
SPINORIAL REPRESENTATION OF SURFACES IN LORENTZIAN HOMOGENEOUS SPACES OF DIMENSION13

(1) There exists $\varphi \in \Gamma(U \Sigma)$ such that

$$
\nabla_{X} \varphi=-\frac{1}{2} \sum_{j=1}^{2} e_{j} \cdot B\left(X, e_{j}\right) \cdot \varphi+\frac{1}{2} X_{0} \cdot \nu \cdot \varphi
$$

for all $X \in T M$ and $X_{0}=X+\left\langle X, e_{0}\right\rangle e_{0}$.

(2) There exists an isometric immersion $F$ of $M$ into $\mathbb{R}_{-} \times \mathbb{S}^{2}$ with second fundamental form $B$.

Moreover, $F: M \longrightarrow \mathbb{R}_{-} \times \mathbb{S}^{2}$ is given by

$$
F=\eta\left\langle\left\langle e_{0} \cdot \varphi, \varphi\right\rangle\right\rangle+\langle\langle\nu \cdot \varphi, \varphi\rangle\rangle .
$$

For the proof we will need the following lemma:

Lemma 6.2. If $\varphi \in \Gamma(U \Sigma)$ is a solution of (45), then, for all $X \in T M$,

$$
\partial_{X}\left\langle\left\langle e_{0} \cdot \varphi, \varphi\right\rangle\right\rangle=0 \text {. }
$$

Proof. We have by definition that

$$
\partial_{X}\left\langle\left\langle e_{0} \cdot \varphi, \varphi\right\rangle\right\rangle=\left\langle\left\langle\nabla_{X} e_{0} \cdot \varphi, \varphi\right\rangle\right\rangle+(i d+\tau)\left\langle\left\langle e_{0} \cdot \nabla_{X} \varphi, \varphi\right\rangle\right\rangle
$$

Following the proof of Proposition 5.2 we find that

$(47)(i d+\tau)\left\langle\left\langle e_{0} \cdot \nabla_{X} \varphi, \varphi\right\rangle\right\rangle=\langle\langle B(X, T) \cdot \varphi, \varphi\rangle\rangle+(i d+\tau)\left\langle\left\langle f N \cdot \nabla_{X} \varphi, \varphi\right\rangle\right\rangle$.

Since $\varphi$ satisfies equation (45) we have that

$(i d+\tau)\left\langle\left\langle f N \cdot \nabla_{X} \varphi, \varphi\right\rangle\right\rangle=\frac{1}{2}(i d+\tau)\left\langle\left\langle f N \cdot\left(-\sum_{j=1}^{2} e_{j} \cdot B\left(X, e_{j}\right) \cdot \varphi+X_{0} \cdot \nu \cdot \varphi\right), \varphi\right\rangle\right\rangle$.

We simplify the above expression replacing

$$
\left\langle\left\langle-\frac{f}{2} N \cdot\left(\sum_{j=1}^{2} e_{j} \cdot B\left(X, e_{j}\right) \cdot \varphi\right), \varphi\right\rangle\right\rangle=-\frac{f}{2}\langle\langle S(X) \cdot \varphi, \varphi\rangle\rangle
$$

which implies

$$
\frac{1}{2}(i d+\tau)\left\langle\left\langle f N \cdot\left(-\sum_{j=1}^{2} e_{j} \cdot B\left(X, e_{j}\right) \cdot \varphi\right), \varphi\right\rangle\right\rangle=-f\langle\langle S(X) \cdot \varphi, \varphi\rangle\rangle .
$$

On the other hand,

$$
\tau\left\langle\left\langle f N \cdot X_{0} \cdot \nu \cdot \varphi, \varphi\right\rangle\right\rangle=-\left\langle\left\langle f N \cdot X_{0} \cdot \nu \cdot \varphi, \varphi\right\rangle\right\rangle
$$

and consequently,

$$
\frac{1}{2}(i d+\tau)\left\langle\left\langle f N \cdot X_{0} \cdot \nu \cdot \varphi, \varphi\right\rangle\right\rangle=0 .
$$

From the equations (47), (48) and (49) it follows that

$$
(i d+\tau)\left\langle\left\langle e_{0} \cdot \nabla_{X} \varphi, \varphi\right\rangle\right\rangle=\langle\langle B(X, T) \cdot \varphi, \varphi\rangle\rangle-f\langle\langle S(X) \cdot \varphi, \varphi\rangle\rangle .
$$

Finally, the conditions (43) and (44) imply that

$$
\nabla_{X} e_{0}=f S(X)+\langle S(X), T\rangle N=f S(X)-B(X, T),
$$

and therefore

$$
\left\langle\left\langle\nabla_{X} e_{0} \cdot \varphi, \varphi\right\rangle\right\rangle+(i d+\tau)\left\langle\left\langle e_{0} \cdot \nabla_{X} \varphi, \varphi\right\rangle\right\rangle=0,
$$

which ends the proof of the lemma. 
The proof of the theorem then relies on the following lemma whose proof will be omitted since it is very similar to the proofs of Lemma 5.1 and Proposition 5.2 (see [28] for more details).

Lemma 6.3. The function $F$ defined by (46) satisfies $d F(X)=\langle\langle X \cdot \varphi, \varphi\rangle\rangle$ for all $X \in T M$.

Sketch of the proof of Theorem 9. Suppose that there exists a solution $\varphi \in \Gamma(U \Sigma)$ of (45), then $F$ defined by (46) takes values in $\mathbb{R}_{-} \times \mathbb{S}^{2}$ : it follows from Lemma 6.2 that $\left\langle\left\langle e_{0} \cdot \varphi, \varphi\right\rangle\right\rangle$ is constant and can be identified after a rigid motion with the first element $e_{1}$ of the standard basis of $\mathbb{R}^{1,3}$; by definition $\langle\langle\nu \cdot \varphi, \varphi\rangle\rangle=\operatorname{Ad}\left([\varphi]^{-1}\right)([\nu])$ with $[\varphi]^{-1} \in \operatorname{Spin}(1,3)$, so that $\langle\langle\nu \cdot \varphi, \varphi\rangle\rangle$ has norm 1; furthermore $\langle\langle\nu \cdot \varphi, \varphi\rangle\rangle$ and $\left\langle\left\langle e_{0} \cdot \varphi, \varphi\right\rangle\right\rangle=e_{1}$ are orthogonal, so $\langle\langle\nu \cdot \varphi, \varphi\rangle\rangle \in \mathbb{S}^{2}$ and $F(M) \subset \mathbb{R}_{-} \times \mathbb{S}^{2}$. By Lemma 6.3 we have that $d F(X)=\langle\langle X \cdot \varphi, \varphi\rangle\rangle$ and we can follow the proof of Proposition 5.2 to obtain that $F: M \longrightarrow \mathbb{R}_{-} \times \mathbb{S}^{2}$ is an isometry and

$$
\begin{aligned}
\Phi_{E}: \quad E & \longrightarrow T\left(\mathbb{R}_{-} \times \mathbb{S}^{2}\right) \\
X \in E_{m} & \longmapsto(F(m),\langle\langle X \cdot \varphi, \varphi\rangle\rangle)
\end{aligned}
$$

is a bundle isomorphism between $E$ and the normal bundle of the immersion $F$ which identifies $B$ to the second fundamental form. The implication $2 \Longrightarrow 1$ is a consequence of the Gauss formula, equation (41).

\section{Representation of Surfaces in $\mathbb{L}(\kappa, \tau)$ Spaces}

In analogy with the Riemannian homogeneous spaces $\mathbb{E}(\kappa, \tau)$ (see [10] and [16]), the 3-dimensional Lorentzian homogeneous spaces $\mathbb{L}(\kappa, \tau)$ are Lorentzian fibrations $\mathbb{L}(\kappa, \tau) \rightarrow M^{2}(\kappa)$ where $M^{2}(\kappa)$ is a Riemannian surface with constant sectional curvature $\kappa$; the fibers of the projection are integral curves of a (complete) timelike unit Killing vector field $\xi$ over the total space and $\tau$ is the bundle curvature which is defined as the real number such that

$$
\bar{\nabla}_{X} \xi=-\tau X \times \xi
$$

where $\bar{\nabla}$ is the Levi-Civita connection and $\times$ is the natural cross product in $\mathbb{L}(\kappa, \tau)$. A precise description of these spaces is the following: for $\kappa \in \mathbb{R}$, defining

$$
V:=\left\{(x, y, z) \in \mathbb{R}^{3}: 1+\frac{\kappa}{4}\left(x^{2}+y^{2}\right)>0\right\} \quad \text { and } \quad \lambda:=\frac{1}{1+\frac{\kappa}{4}\left(x^{2}+y^{2}\right)},
$$

the space is

$$
\mathbb{L}(\kappa, \tau)=\left(V, \lambda^{2}\left(d x^{2}+d y^{2}\right)-(\tau \lambda(y d x-x d y)+d z)^{2}\right) .
$$

Using this representation for $\mathbb{L}(\kappa, \tau)$ and setting $\sigma=\frac{\kappa}{2 \tau}$ the frame

$$
\begin{aligned}
& E_{1}=\lambda^{-1}\left(\cos (\sigma z) \partial_{x}+\operatorname{sen}(\sigma z) \partial_{y}\right)+\tau(x \operatorname{sen}(\sigma z)-y \cos (\sigma z)) \partial_{z} \\
& E_{2}=\lambda^{-1}\left(-\operatorname{sen}(\sigma z) \partial_{x}+\cos (\sigma z) \partial_{y}\right)+\tau(x \cos (\sigma z)-y \operatorname{sen}(\sigma z)) \partial_{z} \\
& E_{3}=\partial_{z}
\end{aligned}
$$

is orthonormal and such that $\left\langle E_{1}, E_{1}\right\rangle=\left\langle E_{2}, E_{2}\right\rangle=1=-\left\langle E_{3}, E_{3}\right\rangle$. Moreover, $E_{1} \times E_{2}=-E_{3}, E_{2} \times E_{3}=E_{1}, E_{1} \times E_{3}=-E_{2}$, and, for $\Gamma_{i j}^{k}:=\left\langle\bar{\nabla}_{E_{i}} E_{j}, E_{k}\right\rangle$

$$
\Gamma_{21}^{3}=\Gamma_{13}^{2}=\tau=-\Gamma_{12}^{3}=-\Gamma_{23}^{1}, \quad \Gamma_{31}^{2}=\sigma+\tau=-\Gamma_{32}^{1}
$$

and

$$
\left[E_{1}, E_{2}\right]=2 \tau E_{3}, \quad\left[E_{2}, E_{3}\right]=\sigma E_{1}, \quad\left[E_{3}, E_{1}\right]=\sigma E_{2} .
$$


We describe in the following table the spaces $\mathbb{L}(\kappa, \tau)$ in terms of the values $\kappa$ and $\tau$.

\begin{tabular}{|c|c|c|c|}
\hline & $\kappa<0$ & $\kappa=0$ & $\kappa>0$ \\
\hline$\tau=0$ & $\mathbb{H}^{2}(\kappa) \times \mathbb{R}_{-}$ & $\mathbb{L}^{3}$ & $\mathbb{S}^{2}(\kappa) \times \mathbb{R}_{-}$ \\
\hline$\tau \neq 0$ & $S L_{2}^{1}$ & $N i l_{3}^{1}$ & $\mathbb{S}_{\text {Berger }}^{3,1}$ \\
\hline
\end{tabular}

Some particular cases occur when $\kappa+4 \tau^{2}=0$ : if $\kappa=\tau=0$ then $\mathbb{L}(\kappa, \tau)=\mathbb{L}^{3}$, and in the other case $\mathbb{L}(\kappa, \tau)=\mathbb{H}_{1}^{3}(\kappa)$ (in the table above this space corresponds to $\widetilde{S L_{2}^{1}}$ ). Let us also note that the space $\mathbb{S}_{1}^{3}$ does not admit any unit Killing vector field and therefore cannot be a $\mathbb{L}(\kappa, \tau)$ space.

We now assume $\tau \neq 0$ and characterize the immersion of a Riemannian surface in $\mathbb{L}(\kappa, \tau)$ using its Lie group structure (see [1] for a similar result in the space $\mathbb{E}(\kappa, \tau))$. The Lie algebra of $\mathbb{L}(\kappa, \tau)$ is $\mathfrak{g}=\mathbb{R}^{3}$ with the Lie bracket given in the canonical basis by

$$
\left[e_{1}^{o}, e_{2}^{o}\right]=2 \tau e_{3}^{o}, \quad\left[e_{2}^{o}, e_{3}^{o}\right]=\sigma e_{1}^{o}, \quad\left[e_{3}^{o}, e_{1}^{o}\right]=\sigma e_{2}^{o} .
$$

The metric in $\mathbb{L}(\kappa, \tau)$ is the left-invariant metric $\langle.,$.$\rangle such that \left(e_{1}^{o}, e_{2}^{o}, e_{3}^{o}\right)$ is an orthonormal basis of $\mathfrak{g}$ satisfying $\left\langle e_{1}^{o}, e_{1}^{o}\right\rangle=\left\langle e_{2}^{o}, e_{2}^{o}\right\rangle=1=-\left\langle e_{3}^{o}, e_{3}^{o}\right\rangle$.

Let $M$ be an orientable Riemannian surface and $S: T M \rightarrow T M$ a symmetric operator. Let us suppose that there exist $T \in \Gamma(T M)$ and $\nu \in C^{\infty}(M)$ such that for all $X \in T M$

$$
\begin{gathered}
|T|^{2}-\nu^{2}=-1, \\
\nabla_{X} T=\nu(S(X)+\tau J(X)), \\
d \nu(X)=\langle S(X)+\tau J(X), T\rangle,
\end{gathered}
$$

where $J: T M \rightarrow T M$ is the rotation of angle $+\pi / 2$ in the tangent planes. The following theorem is similar to Theorem 5 in [1] (and we omit the proof here).

Theorem 10. If $M$ is simply connected, the following statements are equivalent:

(1) There exists $\psi \in \Gamma(\Sigma M)$ solution of

$$
\nabla_{X} \psi=\frac{i}{2} S(X) \cdot \psi-\frac{1}{2}(i \tau X+\langle X, T\rangle(\sigma+2 \tau)(i T+\nu)) \cdot \omega \cdot \psi,
$$

for all $X \in T M$ and such that $\left|\psi^{+}\right|^{2}-\left|\psi^{-}\right|^{2}=1$. Here $\omega=\epsilon_{1} \cdot \epsilon_{2}$ where $\left(\epsilon_{1}, \epsilon_{2}\right)$ is a positively oriented orthonormal basis of $M$.

(2) There exists an isometric immersion of $M$ into $\mathbb{L}(\kappa, \tau)$ with shape operator $S$.

As a consequence of that theorem let us deduce a representation theorem for an immersion in the 3-dimensional anti de Sitter space, when we consider this space as the group of $2 \times 2$ matrices

$$
S U_{2}^{1}=\left\{\left(\begin{array}{cc}
z & \omega \\
\bar{\omega} & \bar{z}
\end{array}\right) \in M_{2}(\mathbb{C}):|z|^{2}-|\omega|^{2}=-1\right\},
$$

whose Lie algebra is

$$
\left\{\left(\begin{array}{cc}
i \lambda & a \\
\bar{a} & -i \lambda
\end{array}\right): a \in \mathbb{C}, \lambda \in \mathbb{R}\right\} .
$$

A basis for this Lie algebra is

$$
E_{1}=\left(\begin{array}{cc}
0 & 1 \\
1 & 0
\end{array}\right), \quad E_{2}=\left(\begin{array}{cc}
0 & -i \\
i & 0
\end{array}\right), \quad E_{3}=\left(\begin{array}{cc}
i & 0 \\
0 & -i
\end{array}\right)
$$


the Lie bracket is determined by

$$
\left[E_{1}, E_{2}\right]=2 E_{3}, \quad\left[E_{2}, E_{3}\right]=-2 E_{1}, \quad\left[E_{3}, E_{1}\right]=-2 E_{2},
$$

and the Lorentzian metric of $S U_{1}^{2}$ is the left-invariant metric such that $\left\langle E_{i}, E_{j}\right\rangle=0$ if $i \neq j$ and $\left\langle E_{1}, E_{1}\right\rangle=\left\langle E_{2}, E_{2}\right\rangle=1=-\left\langle E_{3}, E_{3}\right\rangle$. The non-trivial solution $\kappa=-4$ and $\tau=1$ of $\kappa+4 \tau^{2}=0$ gives that $\mathbb{L}(\kappa, \tau)=S U_{2}^{1}$ and from Theorem 10 we deduce the following representation theorem in this space:

Theorem 11. Let $M$ be a simply connected Riemannian surface and $S: T M \rightarrow$ $T M$ a symmetric operator. Let us suppose that there exist $T \in \Gamma(T M)$ and $\nu \in$ $C^{\infty}(M)$ solutions of (53)-(55) with $\tau=1$. The following statements are equivalent:

(1) There exists $\psi \in \Gamma(\Sigma M)$ solution of

$$
\nabla_{X} \psi=\frac{i}{2} S(X) \cdot \psi-\frac{1}{2} i X \cdot \omega \cdot \psi
$$

for all $X \in T M$ and such that $\left|\psi^{+}\right|^{2}-\left|\psi^{-}\right|^{2}=1$.

(2) There exists an isometric immersion of $M$ into $S U_{2}^{1}$ with shape operator $S$.

\section{Correspondences between CMC surfaces}

8.1. Correspondence between CMC surfaces in $\mathbb{R}^{1,2}$ and in $\mathbb{H}_{1}^{3}$. Let $(M, g)$ be an oriented Riemannian surface and denote by $\langle$,$\rangle the real part of the natural$ Hermitian product in $\Sigma M$. Recall that for $X \in T M$ and $\psi_{1}, \psi_{2}$ sections of $\Sigma M$ we have

$$
\left\langle X \cdot \psi_{1}, X \cdot \psi_{2}\right\rangle=\left\langle\psi_{1}, \psi_{2}\right\rangle \text { if }|X|=1 \quad \text { and } \quad\left\langle X \cdot \psi_{1}, \psi_{2}\right\rangle=-\left\langle\psi_{1}, X \cdot \psi_{2}\right\rangle .
$$

Note that if $\psi=\psi^{+}+\psi^{-}$in the decomposition $\Sigma M=\Sigma^{+} M \oplus \Sigma^{-} M$ then $\langle\psi, \psi\rangle=$ $\left|\psi^{+}\right|^{2}+\left|\psi^{-}\right|^{2}$ and if $\bar{\psi}=\psi^{+}-\psi^{-}$then $\psi$ and $\bar{\psi}$ are related by $i e_{1} \cdot e_{2} \cdot \psi=\bar{\psi}$ for $\left(e_{1}, e_{2}\right)$ a positively oriented orthonormal frame of $T M$ and therefore,

$$
\left\langle\psi, i e_{1} \cdot e_{2} \cdot \psi\right\rangle=\langle\psi, \bar{\psi}\rangle=\left|\psi^{+}\right|^{2}-\left|\psi^{-}\right|^{2} .
$$

Here, we first prove that the Killing-type equation characterizing the immersions of $M$ in the $\mathbb{L}(\kappa, \tau)$ spaces with $\kappa+4 \tau^{2}=0$ (see Theorem 10) is equivalent to its associated Dirac equation and deduce that the immersions in these spaces are also characterized by Dirac equations. Let $H: M \longrightarrow \mathbb{R}$ be a differentiable function and $\tau$ a real number.

Theorem 12. There exists a correspondence between the following data:

(1) A section $\psi$ of $\Sigma M$ with $\left|\psi^{+}\right|^{2}-\left|\psi^{-}\right|^{2}=1$ solution of the Dirac equation

$$
D \psi=-i H \psi+i \tau \omega \cdot \psi \text {. }
$$

(2) A pair $(\psi, S)$, where $S$ is a symmetric operator such that $H=\frac{1}{2} \operatorname{tr} S$ and $\psi \in \Gamma(\Sigma M)$ is a solution of

$$
\nabla_{X} \psi=\frac{i}{2} S(X) \cdot \psi-\frac{i}{2} \tau X \cdot \omega \cdot \psi
$$

for all $X \in T M$ such that $\left|\psi^{+}\right|^{2}-\left|\psi^{-}\right|^{2}=1$.

To prove this theorem we use the following lemma whose proof can be found in [28] (see Lemma 9.1.2). 
SPINORIAL REPRESENTATION OF SURFACES IN LORENTZIAN HOMOGENEOUS SPACES OF DIMENSION2B

Lemma 8.1. If $\psi \in \Gamma(\Sigma M)$ with $\left|\psi^{+}\right|^{2}-\left|\psi^{-}\right|^{2}=1$ satisfies (59) then the operator $S: T M \longrightarrow T M$ given by

$$
\langle S(Y), X\rangle=\frac{2}{|\psi|^{2}}\left(\left\langle i X \cdot \nabla_{Y} \psi, \psi\right\rangle-\frac{\tau}{2} g(X, J Y)|\psi|^{2}\right) \quad X, Y \in T M,
$$

is symmetric and its trace is $H=\frac{1}{2} \operatorname{tr} S$; here $J$ still denotes the rotation of angle $+\pi / 2$ in the tangent planes of $M$.

Proof of Theorem 12. The proof of $(2) \Rightarrow(1)$ readily follows from the definition of the Dirac operator and we omit it. If $\psi \in \Gamma(\Sigma M)$ is a solution of equation (59) with $\left|\psi^{+}\right|^{2}-\left|\psi^{-}\right|^{2}=1$ then $\left(\frac{i}{|\psi|} \psi, \frac{i}{|\psi|} e_{1} \cdot \psi, \frac{i}{|\psi|} e_{2} \cdot \psi, \frac{i}{|\psi|} e_{1} \cdot e_{2} \cdot \psi\right)$ is an orthonormal basis of $\Sigma M$ with respect to $\langle$,$\rangle and therefore we have$

$$
\begin{aligned}
\nabla_{e_{1}} \psi & =\frac{1}{|\psi|^{2}}\left\langle\nabla_{e_{1}} \psi, i \psi\right\rangle i \psi+\frac{1}{|\psi|^{2}}\left\langle\nabla_{e_{1}} \psi, i e_{1} \cdot \psi\right\rangle i e_{1} \cdot \psi \\
& +\frac{1}{|\psi|^{2}}\left\langle\nabla_{e_{1}} \psi, i e_{2} \cdot \psi\right\rangle i e_{2} \cdot \psi+\frac{1}{|\psi|^{2}}\left\langle\nabla_{e_{1}} \psi, i e_{1} \cdot e_{2} \cdot \psi\right\rangle i e_{1} \cdot e_{2} \cdot \psi
\end{aligned}
$$

We first note that, for $k=1,2,\left\langle\nabla_{e_{k}} \psi, i e_{1} \cdot e_{2} \cdot \psi\right\rangle=0$. This is a direct consequence of (58) with $\left|\psi^{+}\right|^{2}-\left|\psi^{-}\right|^{2}=1$ and elementary properties of $\langle.,$.$\rangle . We then deduce$ that $\left\langle\nabla_{e_{1}} \psi, i \psi\right\rangle=0$ using the Dirac equation (59):

$$
\begin{aligned}
\left\langle\nabla_{e_{1}} \psi, i \psi\right\rangle & =\left\langle i H \psi+-i \tau e_{1} \cdot \omega \cdot \psi+e_{1} \cdot \nabla_{e_{1}} \psi, i e_{1} \cdot \psi\right\rangle \\
& =-\left\langle D \psi-e_{1} \cdot \nabla_{e_{1}} \psi, i e_{1} \cdot \psi\right\rangle \\
& =-\left\langle e_{2} \cdot \nabla_{e_{2}} \psi, i e_{1} \cdot \psi\right\rangle \\
& =-\left\langle\nabla_{e_{2}} \psi, i e_{1} \cdot e_{2} \cdot \psi\right\rangle \\
& =0 .
\end{aligned}
$$

So, the expression (62) of $\nabla_{e_{1}} \psi$ reduces to

$$
\nabla_{e_{1}} \psi=\frac{1}{|\psi|^{2}}\left(\left\langle\nabla_{e_{1}} \psi, i e_{1} \cdot \psi\right\rangle i e_{1} \cdot \psi+\left\langle\nabla_{e_{1}} \psi, i e_{2} \cdot \psi\right\rangle i e_{2} \cdot \psi\right)
$$

and from Lemma 8.1 we have that

$$
\begin{aligned}
\nabla_{e_{1}} \psi & =\frac{i}{|\psi|^{2}}\left(\left\langle i e_{1} \cdot \nabla_{e_{1}} \psi, \psi\right\rangle e_{1}+\left\langle i e_{2} \cdot \nabla_{e_{1}} \psi, \psi\right\rangle e_{2}\right) \cdot \psi \\
& =\frac{i}{2}\left(\left\langle S\left(e_{1}\right), e_{1}\right\rangle e_{1}+\left(\left\langle S\left(e_{1}\right), e_{2}\right\rangle+\tau g\left(e_{2}, J\left(e_{1}\right)\right)\right) e_{2}\right) \cdot \psi \\
& =\frac{i}{2} S\left(e_{1}\right) \cdot \psi-\frac{i}{2} \tau e_{1} \cdot e_{1} \cdot e_{2} \cdot \psi \\
& =\frac{i}{2} S\left(e_{1}\right) \cdot \psi-\frac{i}{2} \tau e_{1} \cdot \omega \cdot \psi
\end{aligned}
$$

We prove in a similar way that $\nabla_{e_{2}} \psi=\frac{i}{2} S\left(e_{2}\right) \cdot \psi-\frac{i}{2} \tau e_{2} \cdot \omega \cdot \psi$.

As a consequence of Theorems 10 and 12 we have the following proposition.

Proposition 8.2. If $(M, g)$ is a simply connected Riemmanian surface then the following statements are equivalent:

(1) There exists $\psi \in \Gamma(\Sigma M)$ with $\left|\psi^{+}\right|^{2}-\left|\psi^{-}\right|^{2}=1$ solution of the equation

$$
D \psi=-i H \psi+i \tau \omega \cdot \psi \text {. }
$$

(2) There exists an isometric immersion of mean curvature $H$ of $M$ into $\mathbb{L}(\kappa, \tau)$, where $\kappa+4 \tau^{2}=0$. 
Now we prove that CMC surfaces in $\mathbb{R}^{1,2}$ naturally correspond to CMC surfaces in $\mathbb{H}_{1}^{3}$. Since $\mathbb{R}^{1,2}$ and $\mathbb{H}_{1}^{3}$ are $\mathbb{L}(\kappa, \tau)$ spaces, this correspondence is similar to the Lawson type correspondences in the $\mathbb{E}(\kappa, \tau)$ spaces found by Daniel in [10].

Proposition 8.3. Given an orientable Riemannian surface $(M, g)$ and for each $H_{1} \in(-\infty,-1] \cup[1, \infty)$ there exists a correspondence between the spinor fields $\psi_{1}$ of $\Sigma M$ with $\left|\psi_{1}^{+}\right|^{2}-\left|\psi_{1}^{-}\right|^{2}=1$ which satisfy

$$
D \psi_{1}=-i H_{1} \psi_{1}
$$

and the spinor fields $\psi_{2}$ with $\left|\psi_{2}^{+}\right|^{2}-\left|\psi_{2}^{-}\right|^{2}=1$ which satisfy

$$
D \psi_{2}=-i H_{2} \psi_{2}+i \omega \cdot \psi_{2}
$$

for some $H_{2} \in \mathbb{R}$.

Proof. Let $\psi_{1} \in \Gamma(\Sigma M)$ be a solution of (63) such that $\left|\psi_{1}^{+}\right|^{2}-\left|\psi_{1}^{-}\right|^{2}=1$. If $a=\cos \theta+\operatorname{sen} \theta e_{1} \cdot e_{2}$ and $\psi_{2}:=a \cdot \psi_{1}$ then

$$
D \psi_{2}=-i H_{1} \cos 2 \theta \cdot \psi_{2}+i H_{1} \operatorname{sen} 2 \theta e_{1} \cdot e_{2} \cdot \psi_{2} \text {. }
$$

Taking $\theta \in \mathbb{R}$ such that $\sin 2 \theta=1 / H_{1}$, the last equation is equivalent to

$$
D \psi_{2}=-i\left( \pm \sqrt{H_{1}^{2}-1}\right) \psi_{2}+i \omega \cdot \psi_{2}
$$

which is of the form (64).

As a consequence of Propositions 8.2 and 8.3 we have the following result.

Corollary 3. For $H_{1} \in(-\infty,-1] \cup[1, \infty)$ there exists a correspondence between immersions of mean curvature $H_{1}$ of $M$ in $\mathbb{R}^{1,2}$ and immersions of mean curvature $\pm \sqrt{H_{1}^{2}-1}$ of $M$ in $\mathbb{H}_{1}^{3}$.

8.2. Correspondence between minimal surfaces in $\mathbb{R}^{3}$ and maximal surfaces in $\mathbb{R}^{1,2}$. By [11], a minimal immersion of a Riemannian surface in $\mathbb{R}^{3}$ is characterized by the existence of a spinor field $\psi_{1}$ such that

$$
\left|\psi_{1}^{+}\right|^{2}+\left|\psi_{1}^{-}\right|^{2}=1 \text { and } D \psi_{1}=0
$$

Meanwhile, Proposition 8.2 implies that a maximal immersion of a Riemannian surface in $\mathbb{R}^{1,2}$ is given by a spinor field $\psi_{2}$ such that

$$
\left|\psi_{2}^{+}\right|^{2}-\left|\psi_{2}^{-}\right|^{2}=1 \text { and } D \psi_{2}=0
$$

Proposition 8.4. Let $(M, g)$ be an orientable Riemannian surface. A solution $\psi_{1} \in \Gamma(\Sigma M)$ of $(65)$ such that

$$
\left|\psi_{1}^{+}\right|^{2}-\left|\psi_{1}^{-}\right|^{2}>0
$$

naturally corresponds to a solution $\psi_{2} \in \Gamma(\Sigma \bar{M})$ of $(66)$, where $\Sigma \bar{M}$ is a spinor bundle conformal to $\Sigma M$.

Remark 4. It is well known that the components of $\psi_{1}$ in (65) depend on the Gauss map of $M$ in $\mathbb{R}^{3}$; by explicit calculations, it is easy to show that (67) means that the Gauss map of $M$ takes values in the lower hemisphere of $\mathbb{S}^{2}$.

Proof. Let $\psi_{1} \in \Gamma(\Sigma M)$ be a solution of (65). We define $\psi_{2}$ in terms of $\psi_{1}$ as

$$
\psi_{2}:=\frac{1}{\sqrt{\left|\psi_{1}^{+}\right|^{2}-\left|\psi_{1}^{-}\right|^{2}}} \widetilde{\psi_{1}},
$$


where $\widetilde{\psi_{1}}$ is the spinor field $\psi_{1}$ in the spinor bundle of $M$ associated to the conformal metric $\left(\left|\psi_{1}^{+}\right|^{2}-\left|\psi_{1}^{-}\right|^{2}\right)^{2} g$. Then $\left|\psi_{2}^{+}\right|^{2}-\left|\psi_{2}^{-}\right|^{2}=1$, and the conformal invariance of the Dirac operator (see [5, Pag. 69]) implies (66).

We directly deduce the following corollary:

Corollary 4. There exists a conformal correspondence between minimal surfaces in $\mathbb{R}^{3}$ with Gauss map image in an hemisphere and maximal surfaces in $\mathbb{R}^{1,2}$.

Remark 5. It can be shown by explicit calculations that the correspondence $\psi_{1} \mapsto$ $\psi_{2}$ corresponds to the transformation

$$
\int\left(\Phi_{1}, \Phi_{2}, \Phi_{3}\right) d z \mapsto \int\left(i \Phi_{1}, i \Phi_{2}, \Phi_{3}\right) d z
$$

on Weierstrass data of immersions in $\mathbb{R}^{3}$ and in $\mathbb{R}^{1,2}$ (details are carried out in [28, Sec 9.2]). This is the correspondence described in [15]. We point out that for the general correspondence no hypotheses on the Gauss map image of the minimal surface is needed, see [15].

\section{Appendix A. IDENTIFICATION OF SPINOR BUNDLES}

We consider a simply connected pseudo-Riemmanian surface $M$ and the trivial bundle $E=M \times \mathbb{R} \longrightarrow M$ with metric $\mp d \nu^{2}$ depending on whether $M$ is Riemannian or Lorentzian and let us denote the spin structures of $T M$ and $E$ by $\widetilde{Q}_{M}$ and $\widetilde{Q}_{E}$ respectively. We identify here the spinor bundle $\Sigma M$ with a subbundle of the bundle $\Sigma=\left(\widetilde{Q}_{M} \times{ }_{M} \widetilde{Q}_{E}\right) \times_{\rho} C l_{1,2}$ defined by (6).

Let us begin with the Riemannian case. We define the complexified quaternions as

$$
\mathbb{H}^{\mathbb{C}}:=\mathbb{H} \otimes_{\mathbb{R}} \mathbb{C}=\left\{z_{0}+z_{1} I+z_{2} J+z_{3} K: z_{i} \in \mathbb{C}\right\} .
$$

This algebra is endowed with the quadratic form $\langle q, q\rangle=z_{0}^{2}+z_{1}^{2}+z_{2}^{2}+z_{3}^{2}$, where $q=z_{0}+z_{1} I+z_{2} J+z_{3} K \in \mathbb{H}^{\mathbb{C}}$.

Remark 6. Let $\left(e_{0}, e_{1}, e_{2}\right)$ be the standard basis of $\mathbb{R}^{1,2}$, which is such that $\left\langle e_{i}, e_{j}\right\rangle=$ 0 if $i \neq j$ and $\left\langle e_{0}, e_{0}\right\rangle=-1=-\left\langle e_{1}, e_{1}\right\rangle=-\left\langle e_{2}, e_{2}\right\rangle$. The map obtained by linearity from $e_{0} \longmapsto i I, e_{1} \longmapsto J, e_{2} \longmapsto J I=-K$ is a Clifford application which induces an isomorphism of algebras between $C_{1,2}$ and $\mathbb{H}^{\mathbb{C}}$. Using this isomorphism, the even Clifford algebra $\mathrm{Cl}_{1,2}^{0}$ is isomorphic to

$$
\left\{q_{0}+q_{1} I+i q_{2} J+i q_{3} K: q_{i} \in \mathbb{R}\right\} .
$$

We consider the following representations of the group $\operatorname{Spin}(0,2)$ given by the left-multiplication:

$$
\rho_{1}: \operatorname{Spin}(0,2) \longrightarrow G l\left(C l_{0,2}\right) \quad \text { and } \quad \rho_{2}: \operatorname{Spin}(0,2) \subset \operatorname{Spin}(1,2) \longrightarrow G l\left(C l_{1,2}^{0}\right) .
$$

Here and below we use the models

$$
C l_{0,2}=\mathbb{H} \quad \text { and } \quad \operatorname{Spin}(0,2)=\left\{q_{0}+q_{1} I: q_{i} \in \mathbb{R}, q_{0}^{2}+q_{1}^{2}=1\right\} .
$$

The representation $\rho_{1}$ is the standard spin representation (if we identify $\mathbb{H} \simeq \mathbb{C}^{2}$ ). The following lemma states that the representations $\rho_{1}$ and $\rho_{2}$ are equivalent. 
Lemma A.1. The isomorphism of vector spaces

$$
\begin{aligned}
f: \quad C l_{0,2} & \longrightarrow C l_{1,2}^{0} \\
q_{0}+q_{1} I+J\left(q_{2}-I q_{3}\right) & \longmapsto q_{0}+q_{1} I+i J\left(q_{2}-I q_{3}\right)
\end{aligned}
$$

induces a $\mathbb{C}$-linear isomorphism between the representations $\rho_{1}$ and $\rho_{2}$, where the complex structure in both spaces is given by the right multiplication by $I$.

Proof. A computation shows that $f(g q)=g f(q) \forall g \in \operatorname{Spin}(0,2), \forall q \in C l_{0,2}$.

Identifying $e_{0}$ with $i I$ (see Remark 6), the isomorphism $f$ satisfies the following:

Lemma A.2. If $x \in \mathbb{R}^{2}$ and $q \in \mathbb{H}$ then $f(x \cdot q)=i e_{0} \cdot x \cdot f(q)$, where $i$ is the complex structure in $C l_{1,2}^{0}$ given by the right multiplication by $I$.

Since $E$ is trivial the orthonormal frame bundle is $Q_{E}=M \times\{1\}$ and $\widetilde{Q}_{E}=$ $M \times\{ \pm 1\}$ is also trivial. We consider the global section $\widetilde{s}_{E}: M \longrightarrow\{ \pm 1\}$ given by $m \longmapsto+1$. Considering the inclusion $\widetilde{Q}_{M} \longrightarrow \widetilde{Q}_{M} \times \widetilde{Q}_{E}, \widetilde{s}_{M} \longmapsto\left(\widetilde{s}_{M}, \widetilde{s}_{E}\right)$ and the isomorphism $f$ of Lemma A.2 we get the bundle isomorphism

$$
\begin{aligned}
\Sigma M & :=\widetilde{Q}_{M} \times_{\rho} \Sigma_{2} \longrightarrow \Sigma_{0}:=\left(\widetilde{Q}_{M} \times_{M} \widetilde{Q}_{E}\right) \times_{\rho} C l_{1,2}^{\circ} \\
\psi:=\left[\tilde{s}_{M}, q\right] & \longmapsto \psi^{*}:=\left[\left(\tilde{s}_{M}, \tilde{s}_{E}\right), f(q)\right] ;
\end{aligned}
$$

it satisfies

$$
\left(\nabla_{X} \psi\right)^{*}=\nabla_{X} \psi^{*}, \quad\left(X \cdot_{M} \psi\right)^{*}=i N \cdot X \cdot \psi^{*} \quad \text { and }\left|\psi^{+}\right|^{2}-\left|\psi^{-}\right|^{2}=\left\langle\left\langle\psi^{*}, \psi^{*}\right\rangle\right\rangle
$$

for all $X \in T M$, where $N=\left[\left(\tilde{s}_{M}, \tilde{s}_{E}\right), e_{0}\right]$ (see [28, Prop. 3.4.9] for more details).

Let us see now the Lorentzian case. Here the spinor bundle of $M$ is $\Sigma M=$ $\widetilde{Q}_{M} \times_{\rho_{1}} C l_{1,1}$, where $\rho_{1}: \operatorname{Spin}(1,1) \longrightarrow G l\left(C l_{1,1}\right)$ is the representation given by left-multiplication. As above we have $\widetilde{Q}_{E}=M \times\{ \pm 1\}$, the section $\widetilde{s}_{E}: m \mapsto+1$ of $\widetilde{Q}_{E}$, and the inclusion $\tilde{Q}_{M} \longrightarrow \widetilde{Q}_{M} \times \widetilde{Q}_{E}, \widetilde{s}_{M} \longmapsto\left(\tilde{s}_{M}, \widetilde{s}_{E}\right)$. Using the isomorphism $C l_{1,1} \simeq C l_{1,2}^{0}$ (induced by the Clifford application $\mathbb{R}^{1,1} \longrightarrow C l_{12}^{0}, x \longmapsto x \cdot e_{2}$, where $\left(e_{0}, e_{1}, e_{2}\right)$ is the standard basis of $\left.\mathbb{R}^{1,2}\right)$ we obtain a bundle isomorphism

$$
\begin{aligned}
\tilde{Q}_{M} \times{ }_{\rho_{1}} C l_{11} & \longrightarrow\left(\widetilde{Q}_{M} \times{ }_{M} \tilde{Q}_{E}\right) \times{ }_{\rho} C l_{1,2}^{0} \\
\psi & \longmapsto \psi^{*}
\end{aligned}
$$

it satisfies the properties

$$
\left(\nabla_{X} \psi\right)^{*}=\nabla_{X} \psi^{*}, \quad(X \cdot M \psi)^{*}=X \cdot N \cdot \psi^{*} \quad \text { and } \quad\left|\psi^{+}\right|^{2}-\left|\psi^{-}\right|^{2}=\left\langle\left\langle\psi^{*}, \psi^{*}\right\rangle\right\rangle
$$

for all $X \in T M$, where $N=\left[\left(\tilde{s}_{M}, \tilde{s}_{E}\right), e_{2}\right]$ (see [28, Prop. 3.4.16] for more details).

\section{Appendix B. Bivectors ANd linear operators}

We prove that a skew-symmetric operator $u: \mathbb{R}^{r, s} \longrightarrow \mathbb{R}^{r, s}$ is identified with a bivector $\underline{u} \in \Lambda^{2}\left(\mathbb{R}^{r, s}\right)$. Let us consider the following bracket in the Clifford algebra $C l_{r, s}$

$$
[a, b]=\frac{1}{2}(a \cdot b-b \cdot a)
$$

for all $a, b \in C l_{r, s}$. 
Lemma B.1. Let $u: \mathbb{R}^{r, s} \longrightarrow \mathbb{R}^{r, s}$ be a skew-symmetric operator. The bivector that represents $u$ is

$$
\underline{u}=\frac{1}{2} \sum_{j=1}^{r+s} \varepsilon_{j} e_{j} \cdot u\left(e_{j}\right), \quad \varepsilon_{j}=\left\langle e_{j}, e_{j}\right\rangle= \pm 1,
$$

and for all $\xi \in \mathbb{R}^{r, s}$ we have

$$
[\underline{u}, \xi]=u(\xi) .
$$

Proof. Let us consider the linear application $u: \mathbb{R}^{r, s} \longrightarrow \mathbb{R}^{r, s}$ given by $e_{i} \longmapsto \varepsilon_{j} e_{j}$ and $e_{j} \longmapsto-\varepsilon_{i} e_{i}$ if $i<j$ and $e_{k} \longmapsto 0$ if $k \neq i, j$ which corresponds to $\varepsilon_{i} e_{i} \wedge \varepsilon_{j} e_{j} \in$ $\Lambda^{2} \mathbb{R}^{r, s}$. This map is skew-symmetric and $\underline{u}=\varepsilon_{i} \varepsilon_{j} e_{i} \cdot e_{j}=\frac{1}{2} \varepsilon_{i} \varepsilon_{j}\left(e_{i} \cdot e_{j}-e_{j} \cdot e_{i}\right)$ satisfies

$$
\left[\underline{u}, e_{k}\right]=\frac{1}{2} \varepsilon_{i} \varepsilon_{j}\left(e_{i} \cdot e_{j} \cdot e_{k}-e_{k} \cdot e_{i} \cdot e_{j}\right)
$$

which yields for $k=i$

$$
\left[\underline{u}, e_{i}\right]=\frac{1}{2} \varepsilon_{i} \varepsilon_{j}\left(\varepsilon_{i} e_{j}+\varepsilon_{i} e_{j}\right)=\varepsilon_{j} e_{j}=u\left(e_{i}\right)
$$

similarly we can prove that $\left[\underline{u}, e_{j}\right]=u\left(e_{j}\right)$ and also readily see that $\left[\underline{u}, e_{k}\right]=0$ for $k \neq i, j$. Equality (70) is a consequence of linearity.

Acknowledgments. The author was partially supported by the project PAPIIT IA106218. She thanks P. Bayard for valuable suggestions during the development of this work and J. Roth for useful conversations, especially about the $\mathbb{L}(\kappa, \tau)$ spaces.

\section{REFERENCES}

[1] P. Bayard, On the spinorial representation of spacelike surfaces into 4-dimensional Minkowski space, Journal of Geometry and Physics 74 289-313 (2013).

[2] P. Bayard, M. -A. Lawn, J. Roth, Spinorial representation of submanifolds in Riemannian space forms, Pacific Journal of Mathematics 291:1 51-80 (2017).

[3] P. Bayard, V. Patty, Spinor representation of Lorentzian surfaces in $\mathbb{R}^{2,2}$, Journal of Geometry and Physics 95 (2014) 74-95.

[4] P. Bayard, J. Roth, B. Zavala, Spinorial representation of submanifolds in metric Lie groups, Journal of Geometry and Physics 44:4 (2017) 433-453.

[5] J. P. Bourguignon, O. Hijazi, J. L. Milhorat, A. Moroianu, S. Moroianu, A Spinorial Approach to Riemannian and Conformal Geometry, EMS Monographs in Mathematics.

[6] M. Cahen, M. Parker, Pseudo-Riemannian symmetric spaces, Mem. Am. Math. Soc. 24 (229) (1980) 1-108.

[7] M. Cahen, N. Wallach, Lorentzian symmetric spaces, Bull. Am. Math. Soc. 76:3 (1970) 585591.

[8] G. Calvaruso, Einstein-like metrics on three-dimensional homogeneous Lorentzian manifolds, Geom. Dedicata 127 (2007) 99-119.

[9] G. Calvaruso, Homogeneous structures on three-dimensional Lorentzian manifolds, Journal of Geometry and Physics 57 1279-1291.

[10] B. Daniel, Isometric immersions into 3-dimensional homogeneous manifolds, Comment. Math. Helv. 82:1 (2007).

[11] T. Friedrich, On the Spinor Representation of Surfaces in Euclidean 3-Space, Journal of Geometry and Physics 28 143-157.

[12] M. A. Lawn, Immersions of Lorentzian surfaces in $\mathbb{R}^{2,1}$, Journal of Geometry and Physics 58:6 (2008) 683-700.

[13] M. A. Lawn, doctoral thesis Méthodes spinorielles et géométrie para-complexe et paraquaternionique en théorie des sous-variétés, Université Henri Poincaré - Nancy I D.F.D. Mathématiques (2007). 
[14] M. A. Lawn, J. Roth, Spinorial Characterizations of Surfaces into 3-dimensional PseudoRiemannian Space Forms Math Phys Anal Geom. 14: 185 (2011).

[15] F. J. López, R. López, R. Souam, Maximal surfaces of Riemann type in Lorentz-Minkowski space $\mathbb{L}^{3}$, Michigan Math. J. 47:3 (2000) 469-497.

[16] J. Manzano, On the classification of the Killing submersions and their isometries, Pacific Journal of Mathematics 270 (2014).

[17] P. Malliavin, Géométrie différentielle intrinsèque, Hermann, Paris, 1972.

[18] W. Meeks, J. Pérez, Constant mean curvature surfaces in metric Lie groups. Contemp. Math. $\mathbf{5 7 0}(2012)$.

[19] J. Milnor, Curvatures of left invariant metrics on Lie groups, Adv. Math. 21 (1976) 293-329.

[20] B. Morel, Surfaces in $\mathbb{S}^{3}$ and $\mathbb{H}^{3}$ via spinors, Séminaire de Théorie spectrale et géométrie, Grenoble, 23 (2004-2005) 131-144.

[21] V. Patty, A generalized Weierstrass representation of Lorentzian surfaces in $\mathbb{R}^{2,2}$ and applications, Int. J. of Geometric Methods in Modern Physics 13:6 (2016) 1650074.

[22] J. Roth, Isometric immersions into Lorentzian products, International Journal of Geometric Methods in Modern Physics, World Scientific Publishing 8:6 (2011) 1-22.

[23] J. Roth, Spinorial characterizations of surfaces into three-dimensional homogeneous manifolds, Journal of Geometry and Physics 60 (2010) 1045-1061.

[24] E. Stein, J.Milnor, M. Spivak, R. Wells, J. Mather, Morse Theory, Annals of mathematics studies 51, Princeton University Press, 1963.

[25] J. A. Wolf, Spaces of Constant Curvature, Publish or Perish, Berkeley, CA, 1984.

[26] H. Wu, On the de Rham decomposition theorem, Illinois J.Math. 8 (1964) 291-311.

[27] H. Wu, Holonomy groups of indefinite metrics, Pacific J.Math. 20 (1967) 351-392.

[28] B. Zavala, doctoral thesis Representación espinorial de superficies en espacios homogéneos lorentzianos 3-dimensionales, Facultad de Ciencias, UNAM, 2020.

E-mail address: bzavala@ciencias.unam.mx

FACULTAD DE CIENCIAS, UNIVERSIDAD NACIONAL AUTÓNOMA DE MÉXICO, AV. UNIVERSIDAD 3000, CIRCUITO EXTERIOR S/N, DELEGACIÓN COYOACÁN, C.P. 04510, CIUDAD UNIVERSITARIA, CDMX, MÉXICO. 